\title{
Antonin Artaud sur la scène espagnole (1969-2016)
}

\author{
Antonin Artaud on the Spanish stage (1969-2016)
}

\author{
Anna Corral Fullà \\ Universitat Autònoma de Barcelona \\ ana.corral@uab.cat
}

\begin{abstract}
The influence of Artaud's theories in the world of theatre is, today, an indisputable reality. Indeed, Artaud represents the pillar on which the collective groups of independent theatre arisen in the years 60-70 (Living Theater, etc.) rest; the most innovative theories of renowned theater directors such as Jerzy Grotowski or Peter Brook; the postdramatic theatre (the performance, among others) and the new rank granted to the word in the theatrical representation. At present, Artaud is an inescapable reference in the theatrical field: Artaud and The theatre and the plague; Artaud and Theatre of cruelty; theoretical writings collected in Theatre and its double.

This article aims to address the reception of Artaud on the Spanish theatre stage. We are interested in revealing what representations of Artaud have been privileged - the theatre man, the madman, the poet, the sick ... - and examine the incidence of these performances in the dissemination of the work of Antonin Artaud in Spain.
\end{abstract}

\section{Keywords}

Artaud, theatrical representation, reception, avant-garde theatre.

\section{Resumen}

La influencia de las teorías de Artaud en el mundo del teatro es, a día de hoy, una realidad indiscutible. En efecto, Artaud representa uno de los pilares sobre el que reposan los grupos colectivos de teatro independiente surgidos en los años 60-70 (Living Theater, etc.); las teorías más innovadoras de directores teatrales de renombre como Jerzy Grotowski o Peter Brook; el teatro posdramático (la performance, entre otros) y el nuevo rango otorgado a la palabra en la representación teatral. En la actualidad, Artaud es una referencia ineludible en el ámbito teatral: Artaud y El teatro y la peste; Artaud y El teatro de la crueldad; escritos teóricos recogidos en El teatro y su doble.

Este artículo se propone abordar la recepción de Artaud en la escena teatral española. Nos interesa identificar qué representaciones de Artaud se han privilegiado - el hombre de teatro, el loco, el poeta, el enfermo... - y examinar la incidencia de dichos espectáculos en la divulgación de la obra de Antonin Artaud en España.

\section{Palabras clave}

Artaud, representación teatral, recepción, teatro vanguardista. 


\section{Introduction}

Affirmer qu'Artaud est un référent du théâtre contemporain, c'est dire, à tous les effets, une évidence. Bertold Brecht et Antonin Artaud marquent en effet les deux voies entreprises par le théâtre depuis le début du XXe siècle jusqu'à nos jours, deux approches théâtrales, en principe opposées, mais qui se trouvent parfois entrecroisées dans les spectacles contemporains. L'un prône la distance qui éveille l'œil critique; l'autre, la perte de toute distance qui amène le spectateur à une "participation vitale" et à un renouveau de l'homme (Rancière, 2008: 11). Le théâtre défendu passionnément par Artaud correspond à cette deuxième voie, un théâtre qui "comme la peste est une crise qui se dénoue par la mort ou par la guérison" (Artaud, 1964: 46). L'influence des théories d'Artaud dans le monde du théâtre est donc une réalité indiscutable à l'heure actuelle. On puise dans ses écrits et c'est, en grande partie, sur son héritage que reposent les groupes collectifs de théâtre indépendant nés dans les années 60-70 (comme le Living Theater); quelques théories et expériences novatrices de certains metteurs en scène (tels Jerzy Grotowski ou Peter Brook); le théâtre post-dramatique (la performance, entre autres) et le nouveau rang accordé à la parole ou délittérarisation du théâtre. De nos jours (et depuis longtemps), Artaud est devenu une référence indéniable lorsqu'on parle de théâtre, une dimension de l'auteur qui est bien répandue et visible en Espagne : Artaud et Le théâtre et la peste; Artaud et Le théâtre de la cruauté; des écrits théoriques recueillis dans Le théâtre et son double.

Dans cet article, nous nous donnons pour objectif d'aborder la réception d'Artaud sur la scène espagnole. Il nous intéresse, en particulier, d'identifier les représentations d'Artaud qui ont été privilégiées sur les planches - l'homme de théâtre, le poète, le fou, le visionnaire... - et d'examiner l'incidence que ces spectacles ont eu pour la vulgarisation de l'œuvre d'Antonin Artaud en Espagne. Un premier chapitre tentera de donner un aperçu général de sa réception pour nous centrer, dans un deuxième temps, sur la représentation théâtrale.

\section{Considérations initiales}

La figure et les textes d'Antonin Artaud ont pénétré en Espagne assez tôt, mais de façon souvent limitée et anecdotique. La première manifestation d'Antonin Artaud apparaît, en fait, lors d'une conférence de Louis Aragon tenue à Madrid, à la Residencia de Estudiantes, le 18 avril $1925^{1}$. Cette conférence a été reprise dans La Révolution surréaliste:

Je vous annonce l'avènement d'un dictateur: Antonin Artaud est celui qui s'est jeté à la mer. Il assume aujourd'hui la tâche immense d'entraîner quarante hommes qui veulent l'être vers un abîme inconnu, où s'embrase un grand flambeau, qui ne respectera rien, ni vos écoles, ni vos vies, ni vos plus secrètes pensées. Avec lui, nous nous adressons

1 Corbalán, Pablo. 05/04/1973. “Antonin Artaud, en la estància impossible” in Informaciones, 36. 
au monde, et chacun sera touché, chacun saura ce qu'il a méprisé de divin, ce qu'il a laissé perdre sous sa forme dans une flaque de soleil, chacun saura son ignominie, celle d'abord des grandes puissances intellectuelles, universités, religions, gouvernements, qui se partagent cette terre, et qui dès l'enfance détournent l'homme de soi-même suivant un dessein ténébreusement préétabli².

Telle est la première image donnée d'Artaud en Espagne, celle d'un agitateur qui bouleverse les fondements mêmes de la société. La seconde référence où est cité le nom d'Artaud dans la presse apparaît dans un article consacré au cinéma le 26 octobre 1927 dans le journal La Vanguardia où il est question de la sortie du film de Germaine Dulac (annoncé pour le 9 février 1928), "La Coquille et le Clergyman", où Artaud est présenté comme "poète surréaliste" qui a élaboré un scénario avec Germaine Dulac à partir d'un "rêve étrange".

Dans le domaine de la théorie théâtrale (recherche, revues spécialisées, critique), le premier texte qui aborde la figure d'Antonin Artaud et ses théories sur l'art dramatique est publié en 1932 dans la revue Teatre Català (Vinyes, 1932: 70-71). L'article est signé par le dramaturge Ramon Vinyes, qui suivait de près l'avant-garde théâtrale et qui avait lu le manifeste du Théâtre de la Cruauté, paru un mois avant dans La Nouvelle Revue Française (in Lladó i Vilaseca, 2002: 891). En à peine deux pages ayant comme rubrique Teatre Estranger, il présente et résume le manifeste d'Antonin Artaud qu'il synthétise en trois volets: "Langage de ce nouveau théâtre", qui correspond au début du manifeste; "Technique", du même nom dans le texte d'Artaud; et un troisième volet, "Pièces" (intitulé "Programme" dans le manifeste d'origine), où Artaud affirmait aller mettre en scène, "sans tenir compte du texte" (Artaud, 1964: 153), neuf pièces, parmi lesquelles le Woyzeck de Büchner, un extrait du Zohar ou du Sade entre autres. Vinyes n'aborde que de manière succincte d'autres aspects recueillis dans le manifeste concernant la mise en scène (environ cinq-six pages du texte d'origine) décor, objets, masques, accessoires, costumes, salle... - qu'il résume en une seule phrase: "Dans le 'Théâtre de la Cruauté', on se servira de masques, de grands instruments de musique, d'acteurs, de lumières qui apparaissent en ondes, de costumes liturgiques [...]"33 (Vinyes, 1932: 71). Le dramaturge catalan donne donc un aperçu du manifeste et le fait avec le même esprit que celui que le texte artaudien insuffle. Il clôt l'article avec quelques questions ouvertes, qui ne manquent pas d'humour, à propos de la proposition d'Artaud:

Nous avons tâché, peut-être par cruauté nous aussi, de le résumer [le manifeste], ne serait-ce que de manière sommaire. Ce travail de synthèse a été comme un jeu. Humoriste, le programme du nouveau théâtre? Illuminé, le programme du nouveau théâtre? Fantaisiste? Peu importe, mais il y a là un programme et quelqu'un qui croit avoir un public qui le suit dans ses préoccupations autour du renouveau du théâtre (Vinyes, 1932: 71).

215 juillet 1925. "Fragments d'une conférence" in La Révolution surréaliste, $n^{\circ} 4$, Editions de la Nouvelle Revue Française, 23-24.

3 "En el 'Théâtre de la Cruauté', s'hi faran servir màscares, grans instruments de música, actors, llums portades en ones, vestuaris litúrgics[...]". Traduction française de l'auteure de cet article.

4 "Nosaltres, qui sap si per crueltat també, hem procurat extractar-lo, encara que sigui sumàriament. L'extracta- 
Le premier manifeste du théâtre de la cruauté d'Artaud est parvenu ainsi à se faire connaître dans le milieu théâtral barcelonais, juste un mois après sa parution en France, grâce à l'article de Vinyes dans la revue Teatre Català.

Le deuxième texte en Espagne à traiter l'écriture d'Antonin Artaud appartient à l'écrivain catalan Josep Palau i Fabre, qui avait fait la connaissance de l'auteur à Paris quelques mois avant sa mort. Palau i Fabre présente Artaud sous un angle nouveau, celui du poète et visionnaire, de la folie comme seule issue pour la poésie face au monde: "le poète, pour sauver la poésie du monde, n'a eu d'autre choix que de devenir fou" (Palau, 1948: 10)5. Palau écrit ainsi un bref article de deux pages en catalan intitulé "Antonin Artaud, o la invitació a la follia", publié dans la revue Ariel en 1948, très vite suivi d'une réécriture augmentée en français la même année: “Antonin Artaud ou l'invitation à la folie”. Artaud est, pour Palau i Fabre (1948: 9-10), l'auteur qui poursuit le travail de Rimbaud. Son œuvre suppose, dit-il, une "digestion complète" de celle de Rimbaud. Il n'y a pas de rupture entre l'un et l'autre et La Lettre à la Voyante complète la Lettre du Voyant, ainsi que les Lettres de Rodez complètent Une saison en enfer. Palau i Fabre devient désormais l'un des divulgateurs de l'œuvre d'Artaud en Catalogne.

Comme nous l'avons constaté, avant les années 60, Antonin Artaud est très peu nommé en Espagne. Il n'y a que l'article de Ramon Vinyes, en 1932, celui de Josep Palau i Fabre en 1948 et, quelques mois plus tard, un article de Julio Cortázar ${ }^{7}$ qui annonce la mort de l'auteur français. Néanmoins, l'oubli d'Artaud peut également être extensif à d'autres pays. Même en France, la figure du poète est peu connue jusqu'à presque la moitié du siècle. Comme le souligne David Bradby, "son influence ne dépassa guère les limites d'un cercle étroit d'amis parisiens" (1990: 20). Palau i Fabre, dans la préface qu'il écrit pour Antonin Artaud: la revolta del teatre modern (1976: 10-11), tente d'expliquer ce manque de popularité avant sa mort en 1948. Il souligne, d'une part, le caractère de son œuvre, qui échappe à toute classification, et qui s'éloigne de l'esprit cartésien qui règne en France et, d'autre part, l'expulsion "ignominieuse" du groupe surréaliste qui isole définitivement l'artiste.

David Bradly marque l'année 1944 comme la date à partir de laquelle la popularité d'Artaud ne cesse de croître en France. C'est le moment où l'auteur réédite son recueil d'essais Le théâtre et son double qui, comme affirme Bradby, "le fit connaître au lendemain de la guerre”, et il ajoute: “[...] passé inaperçu lors de sa publication en 1938 [...], il connut le succès que l'on sait" (1990: 21). Une affirmation à nuancer, car justement l'influence d'Artaud

ment ha estat com un joc. Humorista el programa del teatre nou? D'il-luminat el programa del teatre nou? De fantasista? Com sigui però un programa i algú que creu tenir un públic que el segueix en les seves preocupacions de renovació del teatre". Traduction française de l'auteure de cet article.

5 "El poeta, per a salvar la poesia del món, no ha tingut altre remei que tornar-se boig". Traduction française de l'auteure de cet article.

6 Il publie en 1976 un essai en catalan sur Artaud et le théâtre moderne intitulé Antonin Artaud i la revolta del teatre modern.

7 03/05/1948. "Muerte de Antonin Artaud", in Sur, n 16, Buenos Aires. 
dans le champ théâtral est, en fait, moindre et plus tardive dans l'Hexagone que dans d'autres pays en raison des postulats que l'auteur met en place dans son essai, lesquels sont contraires à la tradition théâtrale française fondée sur le texte et la parole (Lázaro Carreter, 1973: 15-16). Ce parti pris était donc “inacceptable en France”, précisément parce que "l'attitude d'Artaud annonçait un nouveau regard sur les moyens et les possibilités du théâtre" (Grotowski in Artaud, 1972: 9-10) ${ }^{9}$. En effet, la découverte du projet artaudien se produit d'abord à l'étranger grâce à la réalisation de plusieurs expériences théâtrales, dites subversives, qui s'étaient inspirées en lui (Martínez Pérez, 1987: 66). Le mythe Artaud atteint ainsi son sommet un peu partout vers la moitié des années 60 quand on commence à être conscients de l'extension de ses théories.

En Espagne, c'est à partir des années 60 qu'apparaissent des textes courts, souvent sous forme de conférences, puis au début des années 70 des traductions commencent à proliférer et des textes plus longs, des essais voient le jour. Dans ce sens, il est loisible de citer la conférence du Dr. Ancochea, "Antonin Artaud o la opacidad sugerente"10 en 1964, dans laquelle il présente Artaud comme exemple de désaliéné de la maladie mentale et de l'artiste. Il en fait un portrait physique et mental et s'intéresse à la façon qu'Artaud avait de se confronter à la maladie en tant que "hérétique-vitale de vivre sa maladie". Selon lui, l'écriture était pour le poète une voie de libération. En 1968, deux autres conférences présentent cette fois-ci le théâtre d'Artaud: "Artaud en el mundo de hoy"11, conférence d'Alberto Miralles (professeur de l'institut du théâtre de Barcelone), et "El teatro como espectáculo"12, conférence de Jaime Salom (auteur de théâtre), centrée sur les auteurs d'avant-gardes Artaud et Brecht. Très vite, les dramaturges prennent la figure d'Artaud comme un référent inéluctable ${ }^{13}$. De nombreuses conférences, colloques et expositions sur Artaud auront lieu au cours des années suivantes en Espagne ${ }^{14}$.

8 "inacceptable en Francia"; "la actitud de Artaud arrojaba una luz completamente nueva sobre los medios y las posibilidades del teatro". Traduction française de l'auteure de cet article.

9 Rappelons qu'Artaud propose en finir avec le théâtre rationaliste de la parole et qu'il défend un théâtre naissant directement de la scène (gestes, mouvements, sons, couleurs, lumières...), une "sorte de création totale" (Artaud, 1964: 143). En fait, toutes les propositions du projet théâtral artaudien visent un théâtre à caractère sacré, lequel, au travers de la cruauté et la violence, produirait une purification chez le spectateur. Ce théâtre, adressé aux sens, aurait un effet cathartique profond: “c'est par la peau qu'on fera rentrer la métaphysique dans les esprits"(ibid., 153).

10 Dans le cadre du cycle "Psicopatología y axiología" à l’Academia de Ciencias Médicas de Barcelona (Asociación de Artes y Letras).

11 La conférence a lieu le 18 novembre 1968 à l' Asociación Condal de Barcelone.

12 La conférence a lieu au siège du Club Mundo à Barcelone.

13 En 1973, une table ronde, ayant pour thème "L'influence d'Antonin Artaud dans le théâtre contemporain", à laquelle participent Palau i Fabre, Pere Planella, Joan de Sagarra et J. Anton Codina, atteste de cet intérêt. La conférence a lieu lors du vingt-cinquième anniversaire de la mort d'Artaud à L'Orfeó de Sants de Barcelone.

14 Entre autres : (1) Palau i Fabre, "La meva concepció del teatre: el temps teatral, la tragèdia, Antonin Artaud", Institut del Teatre de Barcelona, novembre 1991; (2) Congrès à Saragosse sur la vie et l'œuvre d'Artaud (cinéma, conférences et spectacles), en 1991; (3) Colloque International “Antonin Artaud: la lettre en corps. Lectures de Suppôts et Suppliciations", Barcelone, 2010; (4) Ripoll, López-Petit, Pons, Bassas: “Artaud i l'escriptura subversiva", table ronde à la Llibreria Calders, 2015. 
À partir des années 60, Antonin Artaud et ses théories théâtrales font l'objet également de plusieurs articles dans des revues spécialisées de théâtre en Espagne. C'est la découverte massive dans le milieu théâtral d'Antonin Artaud, une époque où la figure controversée du poète, dramaturge et théoricien fait fureur dans le monde entier et se présente comme l'avant-garde théâtrale. La revue Primer Acto ${ }^{15}$ publie plusieurs articles sur Artaud et ses théories dans les numéros 92 (1968); 108 (1969); 148 (1972); et le numéro 159-16016 (1973) lui est entièrement consacré avec un peu plus d'une dizaine de contributions. Les articles autour de la figure d'Artaud apparaissent également dans d'autres revues spécialisées de théâtre. Ainsi, Yorick ${ }^{17}$ publie en 1967 "Antonin Artaud en el teatro, en la vida y en la muerte" d'Enrique Sordo dans le numéro 21 et, en 1969, il publie la première version espagnole de Les Cenci d'Artaud. Outre les revues consacrées au théâtre, les informations sur Artaud se multiplient dans les journaux et périodiques à partir des années 60: comptes rendus sur les traductions inédites de son œuvre en espagnol venant d'être publiées; données sur la parution de biographies ou de livres autour d'Artaud; vulgarisation de ses théories théâtrales, notamment du théâtre de la cruauté; Artaud, sa vie et sa condition; Artaud, l'écrivain et le poète. En effet, comme le souligne Grotowski, "nous sommes à l'ère d'Antonin Artaud" (in Artaud, 1972: 7), ce que l'on aperçoit par la prolifération dans le monde entier des écrits autour de l'auteur (Domenec Font, 1973: 29). Néanmoins, les informations se focalisent surtout autour de deux centres d'intérêt: ses théories théâtrales et la mythification d'Artaud en tant que fou et visionnaire. La figure de l'homme de lettres, l'écrivain et le poète, ne touche que quelques connaisseurs. Depuis lors, Artaud a été, et l'est toujours, l'objet d'études et d'articles dans les revues spécialisées de théâtre ou dans des revues scientifiques académiques.

Pour les expositions et manifestations artistiques, en voici quelques-unes: (1) "La voix de son Maître", auditions des voix de Gérard Philippe, Bruno Ganz, Antonin Artaud et Jean Vilar, Institut de Teatre de Barcelona, avril 1989; (2) "L'última mirada", autoportraits de Giacometti, Bonnard, Bacon et Artaud, MACBA, Barcelone, 1998; (3) "Palau i Fabre: el poeta alquimista", Arts Santa Mònica, 2000, avec une exposition du journal de Palau i Fabre ayant l'intérêt d'intégrer des fragments des dialogues entre Artaud et le poète catalan lors de ses visites à Ivry; (4) "La bellesa del fracàs/ el fracàs de la bellesa", mai 2004, créations de Duchamp, Beys, Mondrian, Artaud, Fundació Miró; (5) “Art i utopia. L'acció restringida”, 900 œuvres d'artistes, parmi lesquels Mallarmé, Apollinaire, Picasso, Duchamp, Artaud... MACBA, 2004; (6) "Pas moi sans eux", collection de photos de Pierre Borhan, Fundació Foto Colectania, Barcelona, juin 2005, à remarquer les portraits d'Artaud réalisés par Denise Colom en 1947; (7) "Pioneras de la fotografía de vanguardia (1920-1940)", Galería de arte Manuel Barbié, Barcelona, 2006, avec des autoportraits et des portraits d'Artaud parmi d'autres; (8) "Un teatro sin teatro", MACBA, Barcelone, 2007, autour de Beckett, Meyerhold, Kantor, Schlemmer, Artaud, entre autres; (9) “Artaud” à La Casa Encendida de Madrid, juin 2009, où sont exposés les dessins et cahiers d'Artaud; (10) "Espectros de Artaud: lenguaje y arte en los años cincuenta", Musée Reina Sofía, Madrid.

15 Primer Acto: Cuadernos de investigación teatral est une prestigieuse revue qui a été fondée en 1957 par José Monleón et dont l'intérêt s'est toujours centré sur la réflexion critique du théâtre international.

16 Les articles parus dans Primer Acto abordent les théories et les manifestes d'Artaud (le théâtre de la cruauté; le théâtre et la peste; le théâtre d'un visionnaire; le théâtre et son double...); la relation du théâtre contemporain avec Artaud; des témoignages; des impressions; vie, esprit et chronologie; ou de nouvelles perspectives telle que "Artaud et la politique". Mais, en général, ce sont ses théories théâtrales qui deviennent le point central du débat.

17 Yorick est une revue emblématique du milieu théâtral fondée en 1965 (jusqu'en 1974) par Gonzalo Pérez de Oleguer et Francesc Jover et dont l'objet était la réflexion sur le théâtre contemporain. 
Nous ne voulons pas terminer ce premier chapitre sans aborder, quoique succinctement, les traductions d'Artaud. Il est important de souligner d'abord que les premières traductions en espagnol se produisent en Amérique du Sud, essentiellement en Argentine, vers la fin des années $50^{18}$. En Espagne, la publication des textes d'Artaud arrive avec une dizaine d'années de retard et c'est dans les années 70 que les traductions commencent à proliférer. En fait, le premier texte traduit date de 1969. Il s'agit de la pièce de théâtre Les Cenci ${ }^{19}$ parue dans la revue Yorick. Il faut préciser ici qu'après la promulgation de la loi sur la presse du 18 mars 1966 de Manuel Fraga Iribarne ${ }^{20}$, la censure préalable des textes est supprimée ${ }^{21}$. Cela supposait l'adoption d'une censure volontaire, c'est-à-dire que les responsables de l'édition devaient publier en conformité aux critères en vigueur en matière de morale, de politique et de religion sous peine d'amendes ou de saisies. Dans ce cas-là, la pièce a été publiée sans entraves, ce qui n'a pas été le cas pour sa représentation postérieure ${ }^{22}$. Cette même année, la revue Primer Acto $^{23}$ fait connaître trois lettres (1931-1932) d'Artaud à Louis Jouvet, où l'auteur prie au metteur en scène de représenter quelques pièces dans le Théâtre Pigalle, dirigé à l'époque par Jouvet. Un an plus tard, l'essai Le Théâtre et son double est publié pour la première fois en Espagne et traduit par Ramon Barnils en catalan ${ }^{24}$. Puis, en 1971, apparaît sur le marché du livre espagnol la traduction de la Lettre à la voyante ${ }^{25}$ et la pièce d'Artaud Jet de Sang voit le jour dans une anthologie de théâtre Dada ${ }^{26}$. En 1972, trois pièces de l'auteur seront encore réunies et publiées dans un volume intitulé Tres piezas $\operatorname{cortas}^{27}$ qui inclut Samouraï, La Pierre philosophale et Il n'y a plus de firmament, ainsi que le prologue de Grotowski "Artaud y el teatro de la crueldad". Si les premières publications d'Artaud en Espagne se produisent, comme le souligne Domenec Font (1972: 5), "sans rime ni raison", il est intéressant pourtant de constater que c'est le théâtre qui intéresse en premier lieu le public en langue espagnole ou catalane. La figure d'Artaud, à partir de ses traductions et de

18 Artaud, A. 1959. El pesa-nervios, seguido por Fragmentos de un diario de infierno. Buenos Aires, Ediciones Mundonuevo. Traduction de G. Guthmann; Artaud, A. 1964. El teatro y su doble. Buenos Aires, Editorial sudamericana. Traduction d'Enrique Alonso et de Francisco Abelenda; etc.

19 Artaud, A. 1969 (mayo). Los Cenci in Yorick, n 35, 35-54. Traduction de Miguel Alcaraz.

20 Ministre de l'Information et du turisme de 1962 à 1969.

21 Cette réforme s'inscrit dans les années 60, une période où le régime tente de donner une image de libéralisation et d'apparente ouverture auprès des nations démocratiques dont dépendait son développement économique (Muñoz Cáliz, 2007: 91).

22 En effet, les compagnies théâtrales devaient encore soumettre les textes aux membres de la Junta de Censura de Obras de Teatro qui autorisaient ou non leur représentation, dans quelles conditions, avec quelles suppressions, etc. La disparition de la censure théâtrale advient, en fait, avec la promulgation du Real Decreto 262/1978 sur la liberté de représentation des spectacles théâtraux (Muñoz Cáliz, 2005, 409).

23 1969. "Artaud a Jouvet" in Primer Acto, n¹08, 10-11.

24 1970. El teatre i el seu doble. Barcelona, Editorial Anagrama.

25 1971. Carta a la vidente. Barcelona, Tusquets Editores. Traduction de Héctor Manjarrez.

26 1971. El chorro de sangre in Teatro Dadá, Aragon, Artaud, Breton, Picabia, Ribemont-Dessaignes, Soupault, Vitrac, Tzara (Renzo et Simonis Ed.). Barcelona, Barral Editores. Traduction de José Escué.

27 Artaud, A. 1972. Tres piezas cortas. Madrid, Editorial Fundamentos. Prologue de Jerzy Grotowski. Traduction de Carlos Manzano. 
ses représentations, sera désormais associée au théâtre en action et à ses théories. Les grands textes d'Artaud, Héliogabale ${ }^{28}$ et Les Tarahumaras ${ }^{29}$, sont traduits en Espagne en 1972 et un an plus tard, Mensajes revolucionarios ${ }^{30}$, une anthologie qui rassemble une suite de conférences et d'articles publiés au Mexique lors de sa visite au pays en 1936. La même année est publié Cinéma ${ }^{31}$ et, en 1975-1976, Lettres de Rodez $I$ et $I I^{32}$. La poésie ne sera tenue en compte que tardivement et de façon très limitée ${ }^{33}$, à partir de fragments ou de textes en prose: El pesa-nervios ${ }^{34}$ en 1976 et Versions d'Antonin Artaud traduit en catalan par Palau i Fabre ${ }^{35}$ en 1977 qui inclut de nombreux poèmes de l'auteur, dont le recueil Ci-gît et Le théâtre de la cruauté $^{36}$. Les années qui suivent, les textes sont peu à peu traduits en anthologies, comme fragments ou textes complets: Van Gogh ou le suicidé de la société ne voit le jour qu'en $1977^{37}$, Artaud le Mômo ${ }^{38}$ en 1979, Lettres à André Breton ${ }^{39}$ en 1977, Nouveaux écrits de Rodez $z^{40}$ en 1980, etc. Mais les écrits d'Artaud intéressent toujours les éditeurs et continuent d'être publiés et réédités jusqu'à nos jours en Espagne. Ainsi, l'année 2018 a vu paraître une nouvelle version de l'Héliogabale ${ }^{41}$, cette fois-ci en langue catalane.

Les revues Yorick et Primer Acto, ainsi que les maisons d'édition, et tout particulièrement Editorial Fundamentos, ont donc joué un rôle de premier ordre pour la réception d'Artaud en Espagne, ce qui ne s'est pas toujours produit dans le calme. En effet, il va sans dire que, pendant la Dictature franquiste, les écrits d'Antonin Artaud sont souvent lus comme

28 Artaud, A. 1972. Heliogábalo o el anarquista coronado. Madrid, Editorial Fundamentos. Traduction de Carlos Manzano.

29 Artaud, A. 1972. Los Tarahumara. Barcelona, Barral Editores. Traduction Carlos Manzano.

30 Artaud, A. 1973. Mensajes revolucionarios. Madrid, Editorial Fundamentos. Traduction de Cristina Vizcaino.

31 Artaud, A. 1973. El Cine. Madrid, Alianza Editorial. Traduction d'Antonio Eceiza.

32 Artaud, A. 1975. Cartas desde Rodez 1. Madrid, Editorial Fundamentos. Traduction de Ramón Font; Artaud, A. 1976. Cartas desde Rodez 2. Madrid, Editorial Fundamentos. Traduction de Fernando Montes.

33 Parfois les revues y contribuent: Triunfo publie un bref texte d'à peine une page (p. 54), le 27 mars 1971, "Rebelión contra la poesía "de "La révolte de la poésie "(Rodez, 1943), qui est présenté comme un "inédit d'Antonin Artaud ". La traduction n'est pas signée.

Triunfo (1946-1982) - revue emblématique d'information théâtrale et cinématographique qui intégrera plus tard des contenus politiques et économiques - a contribué à la diffusion de l'auteur à travers la publication d'articles et la vulgarisation de la parution en Espagne de volumes autour du personnage.

34 Artaud, A. 1976. El pesa-nervios. Madrid, Editorial Visor. Traduction de Marcos Ricardo Barnatán.

35 Artaud, A. 1977. Versions d'Antonin Artaud. Barcelona, Edicions La Magrana, Col-lecció Cristalls, nº 11. Traduction de Josep Palau i Fabre.

36 À ne pas confondre avec les deux manifestes du même titre publiés dans Le théâtre et son double.

37 Artaud,A. 1977. Van Gogh, El suicidado de la Sociedad; Para acabar de una vez con el juicio de Dios. Madrid, Editorial Fundamentos. Traduction de Ramon Font.

En 1979, l'essai est de nouveau publié: Artaud, A. 1979. Van Gogh, El suicidado por la Sociedad. Barcelona, Dilema. Traduction d'Aldo Pelligrini.

38 Artaud, A. 1979. Artaud le Mômo; Aquí yace; La cultura india. Madrid, Editorial Fundamentos. Traduction de Ramón Font.

39 Artaud, A. 1977. Cartas a André Breton. Barcelona-Palma de Mallorca, Calamus Scriptorius. Traduction de Jaume Pomar.

40 Artaud, A. 1980. Cartas desde Rodez 3. Madrid, Editorial Fundamentos. Traduction de Pilar Calvo.

41 Artaud, A. 2018. Heliogàbal o L'anarquista coronat. Barcelona, Edicions Poncianes. Traduction de Xavier Valls. 
manifestes contre l'ordre établi et que leur publication ne se produit pas sans entraves. Il est à noter que bien que les éditeurs ne soient plus obligés de présenter les textes à la censure après la loi de 1966, certains continuent d'en faire la démarche et de soumettre les livres à la consultation pour éviter des saisies ou des dénonciations judiciaires. Ainsi, par exemple, la maison d'édition Fundamentos présente le dossier pour l'édition de Heliogábalo le 22 avril 1971 (exp. 5736/72). Le censeur hésite: d'emblée il dit qu'il n'y a rien pour s’y opposer et qu'on pourrait l'autoriser, mais il change d'avis sur-le-champ, en fonction des nombreuses références sexuelles, et résout, le 13 mai 1972, qu'il vaut mieux procéder avec le silence de l'administration. Cependant, l'éditeur décide de le publier cette même année en prenant ses risques. La publication de Los Tahumara en 1972 par Barral Editores en est un autre exemple. Luis Barral présente le dossier à la censure le 22 avril 1971 qui résout le 6 mai 1972 en autoriser l'édition. Cependant, le livre déjà publié, il est dénoncé par un citoyen et porté devant les tribunaux. Accusé d'un délit contre la religion catholique, le procureur, qui croit y détecter des indices réels de blasphème et d'offense contre celle-ci, demande que Barral soit inhabilité neuf ans pour la fonction publique et l'enseignement. Le procès a lieu le 18 juin 1974 sans pas mal d'obstacles et Barral est enfin acquitté.

Nous n'avons présenté ici qu'une première approximation aux traductions et publications d'Antonin Artaud en Espagne, notre but étant d'offrir un bref aperçu destiné à contextualiser l'ensemble. Il est à noter pourtant que la question mériterait, en effet, une étude à part et approfondie qui tienne compte de tous ces aspects: quelles traductions, dans quelles conditions, quelles suppressions ou modifications effectuées par la censure et dans quelle mesure ces versions ont été révisées et restituées après la dictature dans les rééditions postérieures.

\section{La scène espagnole et Antonin Artaud}

En Espagne ont été mises en scène plusieurs productions où Antonin Artaud était le protagoniste: soit parce que l'on prend des textes de l'auteur pour en faire un spectacle — des pièces de théâtre (rarement) ou des fragments des essais (de théâtre ou autres) —, soit parce que la figure d'Artaud est invoquée sur scène (fréquemment) ou devient un personnage de fiction d'une nouvelle pièce. En tout, nous avons pu trouver et contraster les données de vingt-sept mises en scène de et sur Artaud, ce qui n'exclut pas l'existence d'autres spectacles autour de cet auteur qui ont pu passer inaperçus à cause d'une absence de presse, due sans doute à la portée limitée de ces productions. Nous n'aborderons pas dans cet article l'influence générale d'Artaud chez les metteurs en scène, les auteurs dramatiques et les compagnies de théâtre en Espagne, ce qui aurait supposé un article spécifique. En effet, l'inventaire est long, car son empreinte sur la dramaturgie contemporaine ne peut être contestée à l'heure actuelle: le théâtre panique d'Arrabal, "le plus fidèle de tous ces théâtres infidèles à Artaud" (Torres Monreal in Martínez Pérez, 1987: 76); les subversives mises en scène de 
Víctor García avec la compagnie Núria Espert dans les années 70, les versions de "Genet [Les bonnes] et de Lorca [Yerma] n'auraient pas été possibles sans la lecture de Le théâtre et son

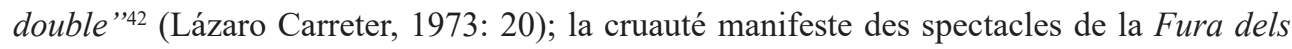
Baus $^{43}$; la violence notoire des pièces d'Angelica Liddel; et un long etcétéra.

Lorsque nous examinons les divers spectacles mis en scène en Espagne autour d'Antonin Artaud, nous constatons au premier abord que ces représentations vont privilégier deux facettes de l'auteur: l'écrivain et l'homme de théâtre — parfois le spectacle même a pour titre l'un de ses essais - et le personnage mythifié, ce dernier permettant l'inclusion de fragments de ses textes littéraires qui facilitent l'identification d'Artaud avec ce fou lucide. Dans tous les cas, le spectacle final se veut avant-gardiste et se présente comme novateur. Ces initiatives viennent pour la plupart de propositions individuelles ou de petites compagnies privées, et les représentations se réalisent, en général, dans des salles de spectacle alternatives à capacité réduite. On peut donc avancer qu'il s'agit d'un théâtre minoritaire adressé à une élite culturelle qui se veut progressiste. Par ailleurs, les différentes représentations autour de la figure d'Antonin Artaud se produisent dans des contextes différents de l'histoire politique et culturelle espagnole : le tardofranquismo (1969-1975), la transition démocratique (19751978) et la démocratie (à partir du 29/12/1978). Si le premier spectacle autour d'Artaud a lieu en 1969 , ce n'est pas par hasard. La dictature touchant déjà à sa $\mathrm{fin}^{44}$, les compagnies de théâtre collectif et indépendant cherchant à réaliser un nouveau théâtre - animées par la liberté politique qui pointait à l'horizon et les mouvements libertaires en Europe à ce

42 "Las versiones de Genet y Lorca no hubieran sido possibles sin la lectura de El teatro y su doble". Traduction française de l'auteure de cet article.

43 L'empreinte d'Artaud se fait sentir dans toute la production de La Fura dels Baus, dont le modus operandi depuis ses premiers spectacles - Accions (1983) ou Suz/o/Suz (1985) - semble s'inspirer tout à fait de l'auteur du théâtre de la cruauté. Dans le Manifeste Canaille du collectif, ils spécifient que "Chaque action représente un exercice pratique, une intervention agressive contre la passivité du spectateur, une sorte de choc dont le but est d'altérer la relation du spectateur au spectacle" (La Fura dels Baus. 2004. La Fura dels Baus 1979-2004. Barcelona, Electa: 30), ce qui renvoie à la conception théâtrale d'Artaud: "une agitation de masses importantes, mais jetées l'une contre l'autre et convulsées, un peu de cette poésie qui est dans les fêtes et dans les foules" (Artaud, 1956-1957: 102). Corral et Lladó mettent justement l'accent sur le caractère rituel de La Fura dels Baus comme base d'une "théâtralité poétique essentielle" (Corral, Anna., Lladó, Jordi. 2014. "Els Joglars et La Fura dels Baus: Corps, musique et danse pour une "métaphysique instantanée" in Quand la scène fait appel... Le théâtre contemporain et le poétique. Paris, L'Harmattan: 160) proche du phénomène des fêtes populaires, un théâtre qui "renonce à tout récit au profit d'une esthétique du présent, de la convulsion/action où se construit, dans un chaos apparent, un riche tissu symbolique" (ibid.: 161), ce qui montre à quel point ce théâtre est débiteur d'Artaud. Les propos d'Artaud se font même entendre dans le spectacle Noun (1990).

Pour le Colloque International “Antonin Artaud, la lettre en corps", la Fura dels Baus avait proposé sur la plage un spectacle original s'inspirant de la poétique artaudienne, le 18 juin 2010.

44 Le tardofranquismo est une période marquée par des conflits sociaux répétés (luttes étudiantes et ouvrières, grèves, activité terroriste de l'ETA, etc.) et par une crise interne au sein du mouvement franquiste (divisé en deux groupes : les technocrates, dits immobilistes, liés à l'Opus Dei, et les partisans pour l'ouverture, aperturistas, associés à la Phalange). L'agitation sociale et le conflit politique se doublent, pourtant, d'une effervescence et d'un renouveau culturels visant la modernité et tentant, en ce qui concerne les arts du spectacle, d'adhérer aux récents et grands courants esthétiques de la représentation théâtrale qui s'imposent en Occident et d'intégrer dans leur production artistique ces nouveaux langages scéniques. 
moment-là (dont Mai 68) - connaissent un essor fulgurant ${ }^{45}$. Ces premières mises en scène appartiennent à une époque glorieuse pleine d'espoir et de découverte, de recherche et de renouveau. A partir du nouveau millénaire, Artaud est revisité régulièrement, mais les propositions scéniques qui voient le jour continuent d'être représentées, à quelques exceptions près, dans des petites salles de théâtre, et de s'adresser au même type de public, cultivé et plutôt intellectuel. Si la période des premières trouvailles est déjà passée, Artaud devient non seulement un "classique anticlassique", mais aussi parfois un personnage de fiction dans de nouveaux spectacles et même dans des romans ${ }^{46}$.

\subsection{Premières approches: les textes d'Artaud}

La première représentation répertoriée date de 1969. Le Teatro Universitario de Cámara de Barcelona (TUC) met en scène Les Cenci d'Artaud. Écrite entre 1927 et 1929, période de la création du Théâtre Alfred Jarry, Les Cenci se centre sur la cruelle histoire de Francesco Cenci. La pièce fut un véritable échec à Paris et ne tint l'affiche qu'à peine 17 jours. En ce qui concerne le spectacle du TUC, la Federación Española de Teatro Universitario présente le dossier à la censure le 14 novembre 1969 et la Junta de Censura Teatral résout le 25 novembre 1969 en autoriser la représentation avec quelques restrictions selon des critères d'ordre moral (sexualité) et religieux. Autorisé uniquement pour des séances de théâtre de chambre "au sens stric" en raison des "relations incestueuses et de l'attaque à la religion et à la famille" (exp. 400/69), la censure limite sa réception et la réduit au petit format, ne pouvant pas atteindre le grand public. Par ailleurs, on soumet également sa représentation au jugement des censeurs le jour de la répétition générale. C'est la période où la censure, s'apercevant du rôle capital des signes scéniques non-verbaux dans le théâtre ${ }^{47}$ de ces années, commence à surveiller de près, non seulement le texte dramatique, mais aussi la mise en scène, apte à accorder un nouveau sens au texte non perceptible à sa lecture (Muñoz Cáliz, 2005: 289). En dernier lieu, le texte dramatique subit aussi une modification avec la

45 Les groupes de théâtre indépendant en Espagne éclosent pourtant dès le début des années 60 et leur activité se développe jusqu'en 1980 environ: Gil Vicente (1961); Teatre experimental català (1962); Els Joglars (1962); Goliardos (1964); Cátaro (1966); Tábano (1968); La Cuadra de Sevilla (1971); Els Comediants (1971); et un long etcétéra.

46 Voici ces deux romans récents: Nieto Foco, Verónica. 2011. La camarera de Artaud. Valladolid, Publicación Diputación de Valladolid. Colección Premios Literarios; Alcoba, Daniel. 2014. Pasión irlandesa de Antonin Artaud. Barcelona, ebookness Editorial.

47 C'est l'éclosion et le développement des groupes de théâtre collectif et indépendant qui accordent de plus en plus d'importance aux différents langages scéniques (gestualité, expression corporelle, costumes, scénographie, musique...). Il s'agit de compagnies qui adoptent une position antifranquiste et qui ont la volonté de changer la société. C'est donc un théâtre à vocation populaire qui tente d'arriver au grand public. Cependant, la censure va entraver la réception de ces spectacles en limitant la représentation à des séances uniques, de théâtre de chambre ou dans les festivals minoritaires (Muñoz, Cáliz, 2005: 292-293), comme on peut l'apprécier avec les restrictions imposées à Les Cenci 
suppression d'une phrase jugée une offense contre la religion ${ }^{48}$. Dans ces conditions et avec ces restrictions, le spectacle a donc pu être représenté.

Le choix de la pièce par le TUC est déjà une déclaration de principes, la compagnie se situant dans la lignée d'un théâtre anticlassique, de rupture, poétique et bouleversant. Le TUC présente son spectacle au Festival Palma - 1969 de Teatro Universitario à Majorque, où ils ont d'ailleurs remporté le prix à la meilleure actrice dans le rôle de Béatrice (Informaciones, 20/04/1970: 27). Ensuite, la pièce se représente au Teatro Marquina de Madrid le 19 avril 1970 dans le cadre du programme du Teatro Nacional de Cámara y Ensayo (TNCE), dirigé par Mario Antolín. Le TNCE commence alors une étape de promotion de nouvelles propositions scéniques et Les Cenci ouvre la saison. Sur le programme, seuls apparaissent le nom de l'auteur, Antonin Artaud, et celui du traducteur, Miguel Alcaraz ${ }^{49}$, le TUC se présentant ainsi comme un groupe de théâtre collectif sans hiérarchie ni proéminence individuelle; un oiseau en cage $e^{50}$, symbole de la troupe, remplace les acteurs à la fin du spectacle au moment de saluer le public. La critique fut, en général, très favorable. On loue la direction scénique et le jeu des acteurs spécialement (Picazo, 21/04/1970: 21; Arriba, 21/04/1970: 18; Informaciones, 20/04/1970: 27; Marquerie, 20/04/1970) $)^{51}$, la critique de Alfredo Marquerie étant à ce propos la plus élaborée:

[Le TUC] a donné à la pièce, dans le rituel et la cérémonie, plasticité et voix chorale, discipline corporelle et même acrobatique, musique de fond et accents justes, compositions de groupes et de figures, emplacements et déplacements, et un jeu d'acteurs rythmique, statique et dynamique, que nous pouvons vraiment qualifier d'exemplaire et d'admirable. [...] Tous [...] depuis les premiers rôles jusqu'aux éléments du chœur méritent de chaleureux et particuliers éloges. De même pour la régie lumière, la plasticité des images et la réalisation scénique (Marquerie, 20/04/1970) ${ }^{52}$.

Un bref article, non signé, paru dans la revue Yorick 38 (1970) signale pourtant un déséquilibre considérable dans la qualité du jeu des acteurs, avec une mention spéciale pour Maite Soler, qui est complimentée. Par ailleurs, la chronique met en valeur, dans le même sens de la citation de Marquerie ci-dessus, la large mise en pratique des théories répandues

48 "Es con el cinismo como se han hecho los papas" (exp. 400/69).

49 Selon Massip (in García Lorenzo, 1999: 131), l'acteur Pepe Rubianes avait participé au montage.

50 Une symbolique ouverte à plusieurs interprétations.

51 La chronique de Lorenzo López Sancho est pourtant moins enthousiaste: "une interprétation émue, sincère [...] avec certaines déficiences pardonnables" ("Interpretación emocionada, sincera [...] con deficiencias perdonables") et "on ne parvient pas pendant la représentation à donner plus de place à la plasticité, aux images [...] qu'à la parole [...], ce qu'on remarque au fil de la représentation" ("no se consigue en la representación dar más valor a la plástica, a lo visual [...] que a la palabra [...] y eso se advierte a lo largo de la representación") (ABC, 22/04/1970: 91). Traduction française de l'auteure de cet article.

52 "[El TUC] dio a la obra, con rito y ceremonia, plástica y voz coral, disciplina corporal e incluso acrobática, música de fondo y acentos justos, composiciones de grupos y figuras, emplazamientos, desplazamientos, rítmica, estática y dinámica interpretación que con justicia podemos calificar de ejemplar y admirable. [...]. Todos [...], desde los primeros papeles a los elementos del coro, merecen encendida y particular alabanza. Y lo mismo la luminoplastia y la realización escénica". Traduction française de l'auteure de cet article. 
sur la cruauté dans le spectacle et, en particulier, le travail d'expressivité corporelle mis en œuvre, devenant l'axe central du montage (1970: 64).

Si la critique admire le choix de la pièce et le montage du TUC, la représentation bénéficie également d'un chaleureux accueil de la part du public et le spectacle est largement applaudi. Quant à la traduction, elle est qualifiée de "correcte" (López Sancho, 22/04/1970: 91), mais on lui attribue parfois "quelques défauts de langage" (Arriba, 21/04/1970: 18). L'initiative du TUC doit être louée et reconnue; ils ont donné à connaître un auteur et une pièce, et ils l'ont fait avec la volonté de suivre les principes théoriques d'Artaud lui-même.

En 1971, une information dans La Vanguardia (12/05/1971: 55) annonce que le comité d'évaluation de la Dirección General de Cultura Popular y Espectáculos ${ }^{53}$ autorise, pour un public de plus de 18 ans, la représentation de La piedra filosofal [La Pierre philosophale] (1931) et Ya no hay firmamento [Il n'y a plus de firmament] (1931-1932) d'Antonin Artaud dans les résidences universitaires ${ }^{54}$ (Colegios Mayores). Cependant, cette nouvelle parue dans les journaux est en contradiction avec le rapport élaboré par les membres de la censure théâtrale ${ }^{55}$. En fait, la compagnie de théâtre Taular-12 présente le dossier à la censure qui résout (exp. 130/71) au mois de mars 1971 interdire la représentation de La Pierre philosophale et autoriser celle d'Il n'y a plus de firmament. Les trois censeurs, Florencio Martínez Ruiz, le religieux Jesús Cea et Antonio de Zubiaurre, coïncident dans leur rapport; l'insolence érotique de La Pierre philosophale devenant l'argument de poids pour son interdiction. En ce qui concerne Il n'y a plus de firmament, ils autorisent la représentation avec plusieurs restrictions ${ }^{56}$ : uniquement pour des séances dans les résidences universitaires (Colegios Mayores ${ }^{57}$ ) de Madrid; pour les plus de 18 ans; et soumise au jugement des censeurs le jour de la répétition générale. Le texte, traduction de Carlos Manzano, subit, en outre, de nombreuses modifications et suppressions concernant le lexique grossier, la morale et la politique $^{58}$. D'ailleurs, les ratures sont appliquées parfois sur les indications scéniques faisant défaut à la bienséance et relevant du mauvais goût ${ }^{59}$. Néanmoins, le rapport final envoyé à la compagnie est rédigé d'une telle manière qu'il se prête à des ambigüités et contradictions : la première page autorise la représentation des deux pièces; la deuxième, avec les indications des censeurs (suppressions, etc.), explicite l'interdiction de La Pierre philosophale. Nous

53 Pendant la dictature de Franco, organisme qui s'occupait de la censure.

54 À l'époque, on parle de Colegios Mayores. Il s'agissait d'une sorte de résidence universitaire qui offrait également des activités culturelles, académiques ou sportives.

55 Junta de Censura Teatral.

56 Avec l'éntrée de Sánchez Bella au Ministère de l'Information et du Tourisme (69-73), il se produit un endurcissement de la censure (Muñoz Cáliz, 2005: 275-278).

57 Selon, Luciano García Lorenzo (1999: 130-131), vers la fin des années 60, le théâtre universitaire arrive à survivre dans quelques-unes des résidences universitaires (Colegios Mayores), car les T.E.U.S des facultés (compagnies de théâtre espagnol universitaire) commencent à décliner dans les années 60 avec l'éclosion du théâtre indépendant.

58 Le mot revolución est à plusieurs reprises supprimé (exp. 130/71).

59 Par exemple, la suppression de la didascalie "pronuncia un discurso hablado, cuyos finales de frase se prolongan en eco y rematan en coros que terminan a su vez en insoportables barridos" (ibid.). 
n'avons pas d'autres données concernant cette représentation, à cause, sans doute, d'une programmation qui restait circonscrite aux murs de ces établissements et donc à portée limitée. Mais il se peut aussi qu'elle n'ait pas eu lieu finalement, car le spectacle envisagé par la troupe Taular-12 rassemblait les deux textes dans un seul spectacle intitulé Répétition générale pour un spectacle d'Antonin Artaud (exp. 130/71). Une énigme reste à propos de cette représentation. Et si jamais elle a vu le jour, quels textes enfin ont été mis en scène?

Entre 1976 et 1989, période qui englobe déjà la transition espagnole et la démocratie, nous avons pu trouver la trace de six spectacles. Le premier, en 1976, est créé par la troupe La Gàbia Teatre ${ }^{60}$ de Vic qui présente Fragments d'un diari d'infern [Fragments d'un journal d'enfer] (répertorié dans Rubio, Carme., 2002: 228) avec l'acteur Ramon Vila à partir du texte d'Artaud du même titre; un spectacle qui est conçu comme un monologue. Quatre ans plus tard, en 1980, Saragosse offre un spectacle créé par l'atelier de recherche théâtrale de Montpellier - de Georges Baal et Gérard Nauret - intitulé Yo soy un rebelde del cuerpo ${ }^{61}$ (vers tiré de l'un des poèmes de l'auteur). Il s'agit d'un “oratorio pour des voix, un trombone, des corps et des cris" (Artal, Rosa María., 21/05/1980) ${ }^{62}$. Le spectacle partait des théories théâtrales d'Artaud et mettait en place ce langage spécifique de la scène que l'auteur avait imaginé: des gestes, des masques, des sons, des mots, des jeux de lumière. Nous ne disposons d'aucune critique de ces deux spectacles: le premier étant simplement répertorié et le dernier, à peine annoncé dans le journal El País, ne compte qu'avec les propos enthousiastes de Rosa María Artal qui affirmait "[ce montage] est un réflexe fidèle de l'œuvre d'Antonin Artaud" (ibid. $)^{63}$. Le troisième spectacle de cette période a lieu au Teatro Español de Madrid le 9 juin 1982 et prend pour titre Heliogábalo. Comme dans le cas précédent, il s'agit d'une production étrangère, italienne plus précisément, avec la troupe La Maschera de Rome et le metteur en scène Meme Perlini. L'Heliogábalo présenté au Teatro Español rassemblait des textes d'Héliogabale ou l'anarchiste couronné d'Artaud et des fragments de Ma mère de Bataille, croisant ainsi deux histoires et deux discours avec dix acteurs sur scène. Selon la critique parue à l' $A B C$, qui n'est pas signée, il s'agit d'un spectacle se caractérisant par son caractère fragmentaire et par une mise en scène proche de l'expressionnisme dotée d" "une lumière qui traverse horizontalement les visages et qui fait ressortir les gestes, en soulignant l'horreur ou le sarcasme" (Interino, 11/06/1982: 69) ${ }^{64}$. Le chroniqueur met l'accent sur la laideur recherchée par ses créateurs dans un montage qui "ne dissimule pas une sale écriture [...], sans être

60 La Gàbia Teatre était une troupe professionnelle catalane, fondée par Lluís Solà, qui s'est définie par la création ou mise en scène d'un type de théâtre d'avant-garde.

61 "Je suis un insurgé du corps", mise en scène de Georges Baal. Représenté au Théâtre Municipal de Montpellier le 25/10/1979 et à l'espace Marais de Paris le 08/12/1980. Information tirée de data.bnf.fr, Bibliothèque Nationale de France.

62 "oratorio para voces, trombón, cuerpos y gritos". Traduction française de l'auteure de cet article.

63 "[...] es un fiel reflejo de la obra de Antonin Artaud” (ibid.). Traduction française de l'auteure de cet article.

64 "la luz barriendo en horizontal los rostros, resaltando los gestos, poniendo acento de terror o de sarcasmo". Traduction française de l'auteure de cet article. 
parvenu à atteindre la lucidité et la violence qui apparaît suggérée dans les textes" (ibid. $)^{65}$. Le journaliste, sur un ton quelque peu acide, n'ôte pas cependant toute la valeur au spectacle, lequel, "depuis notre chronique indigence" (ibid.) ${ }^{66}$, est bien nécessaire et représente une ouverture vers le théâtre européen et la recherche théâtrale.

En 1985, le Teatro Corsario présente à Valladolid une production inspirée du discours radiophonique d'Antonin Artaud Pour en finir avec le jugement de Dieu, enregistré en novembre 1947. Le spectacle, du même nom que la création radiophonique, Para terminar con el juicio de Dios, est réalisé avec la collaboration du groupe musical Qloaca Letal. La représentation a lieu dans une ancienne prison de la ville, un espace à échos artaudiens. Selon le critique Valiño (20/12/1985: 54), le montage était de qualité irrégulière; des fragments très réussis se combinant à d'autres plus pauvres et l'emplacement privilégié de la prison n'était pas, selon lui, exploité à son maximum. Par ailleurs, le travail des acteurs et celui des musiciens n'était pas bien ajusté. Comme dans le cas précédent, la critique considère le spectacle comme nécessaire et intéressant, puisqu'il s'agit de faire connaître un auteur et une autre façon de penser et de faire le théâtre, mais affirme que la proposition scénique manque de rigueur.

La Gàbia Teatre de Vic revient sur Artaud une fois de plus en 1987 avec la production Un somni per a Artaud [Un rêve pour Artaud], spectacle en langue catalane comme celui réalisé en 1976 (cf. supra). Il est présenté au Teatre Monumental de Mataró le 5 novembre pour la Mostra d'art experimental (Dalmau, 05/11/1987: 24), qui prend cette fois-ci la dénomination de Matar, oh $!^{67}$ La traduction des textes appartenait à Palau i Fabre et l'adaptation et la mise en scène à Joan Anguera. Il s'agissait d'une série d'improvisations ${ }^{68}$ à partir de textes d'Artaud dans un espace en rénovation choisi par la compagnie. Ce théâtre en travaux, présentant un aspect quelque peu délabré, leur paraissait approprié pour une mise en scène en rapport avec le théâtre de la cruauté d'Artaud. Pour ce qui est de la représentation, on projetait sur l'un des murs quelques photos d'Artaud et les interprètes jouaient leurs rôles au milieu d'un cercle composé par les spectateurs (une centaine) sur le parterre vide du théâtre en construction. Selon le célèbre chroniqueur de théâtre Joan de Sagarra, Un somni per a Artaud présente des images conventionnelles et fort usées autour de la figure d'Artaud. De plus, dans le spectacle, les mots de l'auteur "semblent, je répète, étranges et, dans la plupart des cas, ils sonnent faux à nos oreilles" (De Sagarra, 07/11/1987: 34) ${ }^{69}$. Néanmoins, il ap-

65 “[...] no disimula una caligrafía sucia [...], sin haber conseguido llegar a la lucidez y a la violencia que aparece sugerida en los textos". Traduction française de l'auteure de cet article.

66 "[...] desde nuestra crónica indigencia". Le journaliste fait référence à l'état précaire du théâtre espagnol de l'époque.

67 Le nom de cet événement théâtral, Matar oh!, faisait un jeu de mots (matar, c'est tuer en espagnol) avec le nom de la ville de Mataró et introduisait, en même temps, le sujet: la mort, point de départ de tous les spectacles du festival (Anguera, Joan, 1996: 31-35).

68 Joan Anguera a qualifié le spectacle, à posteriori, d'une sorte d'action performative (ibid.).

69 "[...] las palabras de Artaud suenan, repito, extrañas y, en la mayoría de los casos, suenan mal". Traduction française de l'auteure de cet article. 
plaudit l'initiative, mais demande un travail sérieux et un effort supplémentaire à la hauteur de La Gàbia Teatre. En fait, la pièce avait été montée en 10 jours, un temps insuffisant pour un projet aussi ambitieux.

La dernière production que nous allons aborder dans ce sous-chapitre appartient à la troupe Cambaleo Teatro. Artaud est pourtant invoqué indirectement à travers son discours sur Vincent Van Gogh. En 1989, le groupe Cambaleo Teatro, dirigé par Carlos Sarrió, propose Proyecto Van Gogh, dont la première se tient au Teatro Albéniz de Madrid ${ }^{70}$. Si cette production tourne autour du peintre Vincent Van Gogh, le texte de la pièce, dont l'auteur est Antonio Fernández Lera, prend appui sur des lettres de Van Gogh à son frère Theo, mais aussi sur l'essai d'Artaud Van Gogh, le suicidé de la société. Ces deux discours entrecroisés permettent de montrer, d'une part, la lutte solitaire de l'artiste "pour protéger ses valeurs [...]" et, d'autre part, "le processus d'intégration et de désintégration de la personnalité" (Artezblai, $04 / 11 / 2009)^{71}$. L'initiative sera reprise par la compagnie, avec des modifications profondes, vingt ans plus tard ${ }^{72}$.

Il est à noter qu'à partir de 1969 il se produit en Espagne un foisonnement de spectacles fondés sur les textes d'Artaud. C'est, en premier lieu, les pièces de théâtre qui intéressent les metteurs en scène, mais on y renonce bientôt pour se centrer ensuite sur les grands œuvres de l'auteur, Héliogabale ou Van Gogh, le suicidé de la société, et sur des textes controversés tel Pour en finir avec le jugement de Dieu. Ce phénomène se manifeste également dans d'autres pays d'Occident dans les années 60. En 1964, le célèbre metteur en scène Peter Brook fonde en Angleterre un "Théâtre de la cruauté" pour voir "si l'incarnation égalait en force l'évocation" (Kustow, 1965: 958) que l'on trouve dans Le théâtre et son double. Le projet contenait une expérience avec la pièce Le jeu de sang qui n'a pas été bien accueillie et Brook a continué sa recherche sur Artaud en appliquant sa méthode à des textes dramatiques contemporains (Martínez Pérez, 1987: 69) ${ }^{73}$. En Espagne, les productions qui ont eu lieu de 1969 à 1989 illustrent un premier regard sur l'œuvre et la figure d'Antonin Artaud. Il y a toujours la volonté de faire connaître la pensée subversive de l'auteur à partir de la mise en scène de ses textes et de la matérialiser en suivant les principes qu'Artaud avait tellement défendus, même si parfois la métaphysique tant recherchée par l'auteur risque de devenir pure esthétique de la laideur. Et si les compagnies qui ont créé ces spectacles sont à louer pour avoir mis ces textes et cet auteur sur les planches, le donnant ainsi à connaître, la qualité des spectacles abordés manque, en général, de rigueur et le résultat pèche d'un certain simplisme. C'est un défaut de jeunesse sans doute, mais leurs propositions semblent mettre en place une esthétique artaudienne "apprise", composée de cris, de gestes, de voix et de

70 Le spectacle voyage ensuite à Varsovie et Gdansk le 28 septembre et les 3 et 4 octobre.

71 "para proteger sus valores [...]" y "el proceso de integración y desintegración de la personalidad". Traduction française de l'auteure de cet article.

72 La deuxième version, intitulée Proyecto Van Gogh: entre los paisajes, est représentée dans la Nave de Cambaleo de Aranjuez (Madrid) en 2010.

73 Kustow (1965: 959) affirme même que certains des textes, joués devant le public, manquaient leur effet. 
corps, mais dont le fond serait encore à trouver, à découvrir. Ou comme le disait De Sagarra dans sa chronique, il leur faudrait un travail sérieux qui les amènerait à la création d'images “qui sortiraient directement de ce ventre qui, selon les mots d'Artaud, évoque la table d'opérations et la morgue" ${ }^{\prime 74}$ (op. cit.).

\subsection{Modification de la perspective: la folie et la lucidité d'Artaud}

Nous avons réuni dans ce sous-chapitre les spectacles produits entre 1991 et 2005. Il s'agit, dans la plupart, de productions qui mettent en scène Artaud lui-même en tant que personnage et qui abordent une période de sa biographie généralement en rapport avec son état mental. Certes, l'intérêt pour ses textes se maintient, mais l'optique change; c'est la période de la construction du personnage Artaud.

Le premier spectacle ayant ces caractéristiques arrive de la main de la jeune compagnie Spitteatre, qui présente en 1991 Artaud! El teatre de la crueltat. La première officielle se déroule le 21 juin au Casal Pere Quart de Sabadell (Barcelone) avec un public réduit. Le spectacle avait déjà été représenté en avant-première au bar l'Espanyol le 8 novembre 1990 et avait reçu un très bon accueil de la part des assistants (Sabadell, 20/06/1991: 37). Cette fois-ci, il est question d'un monologue en catalan créé et interprété par Ramon Costa à partir de textes de l'auteur; direction artistique de Santi Miquel et scénographie de Paco Vigueras et Cesc Arbós. Cette proposition scénique met en scène Antonin Artaud lui-même à sa sortie de Rodez et lors de sa conférence sur la scène du Vieux-Colombier le 13 janvier 1947. Il s'agit d'une tentative de rapprochement de l'auteur: de sa vie et de son œuvre. En outre, le spectacle se veut héritier du théâtre de la cruauté d'Artaud et essaie d'éveiller le public, lequel est directement interpellé et blâmé. Le spectacle envisage de mettre en place certaines des théories théâtrales de l'auteur et déploie largement des éléments plastiques et visuels, sonores et même olfactifs. Selon Cascón i Ramoneda, le spectateur est confronté à une expérience théâtrale, car on ne peut parler proprement de pièce de théâtre (25/06/1991: 32). Cette proposition scénique part d'une recherche préalable sur la figure et l'œuvre d'Artaud et contribue à diffuser un type de théâtre non conventionnel dont le besoin se fait encore sentir dans les années 90 en Espagne. Comme le souligne le chroniqueur de théâtre, il faut mettre en valeur "la tentative honnête de faire un théâtre cruellement bouleversant en marge des poussées institutionnelles"75 (ibid.). Ramon Costa reprendra ce monologue à plusieurs reprises: par exemple, en 1995, il le présente au Centre Industrial de Sabadell et le 22 mai 2004 le spectacle est représenté au Casino (Diari de Sabadell, 13/05/2004: 25) de la ville de Vic avec quelques modifications.

74 "que saliesen directamente de ese vientre que, en palabras de Artaud, evoca la mesa de operaciones y el depósito de cadáveres". Traduction française de l'auteure de cet article.

75 “[...]l'intent honest de fer un teatre cruelment impactant al marge de les empentes institucionals". Traduction française de l'auteure de cet article. 
Le 31 octobre 1992, à la Sala Triángulo ${ }^{76}$ de Madrid, dans le cadre de la IV Muestra Alternativa del Festival de Otoño, a lieu la représentation de Van Gogh: el suicidado de la sociedad, avec une mise en scène de Julián Arranz et Rafael Magano et interprétée par ce dernier. Le monologue se situe dans la même lignée que la plupart des productions abordées jusqu'à présent. D'une part, on met en scène et dramatise ce lucide essai de l'auteur où il nous fait part de ses impressions sur Van Gogh, la folie et la société et, d'autre part, la proposition scénique prétend matérialiser la conception théâtrale de l'auteur (Villán, 06/11/1992: 48). Sur scène, un décor simple et évocateur, avec très peu d'éléments, et un corps, avec ses gestes et ses mouvements, qui s'impose et qui récite le texte. Le chroniqueur de théâtre Alberto de la Hera loue cette proposition scénique qui fait preuve, selon lui, d'une forte maîtrise et habileté: "un spectacle très simple, très élémentaire, mais qui ne manque ni de pouvoir de suggestion, ni de capacité de communication" (13/02/93) ${ }^{77}$. En 2001, lors du Festival Grec de Barcelone, l'essai Van Gogh ou le suicidé de la société devient encore le protagoniste le 2 juillet, au Convent de Sant Agustí. Georges Lavaudant en fait une lecture dramatisée, qui est très bien accueillie et louée par la presse, mais qui ne jouit que d'une faible affluence de public (Benach, 04/07/2001: 43).

Nous trouvons encore d'autres spectacles dont le but et la conception formelle mise en œuvre sont similaires. Entre 1994 et 2001: Artaud: retrato feroz (1994); Nanaqui (1998) et Sueños fugaces (2000) entre autres. Le premier a inauguré le Teatro de la Espada de Madera à Madrid en 1994, puis il a été représenté au Festival de Teatre Experimental de Sitges en 1995 et, enfin, au Festival Grec de Barcelone en 1996. Créé par l'argentin Jorge Gorosco, qui jouait cette même pièce depuis vingt ans, le spectacle réunissait différents textes d'Artaud pour présenter une particulière biographie de l'auteur en adoptant une approche théorique antithéâtrale (Torres, 07/10/1994): “La seule possibilité pour qu'Artaud apparaisse, c'est justement la non interprétation, je n'essaie pas d'adapter sa pensée, ses textes, je m'en tiens strictement à son écriture, à la férocité de son écriture" 78 (Jorge Gorosco in ibid.). On peut parler, de nouveau, d'expérience théâtrale à caractère rituel plutôt que de pièce ou de spectacle. Comme l'affirme très justement Rosana Torres, Artaud: retrato feroz était devenu un classique du théâtre alternatif. En ce qui concerne Nanaqui, il est question d'une production chilienne de Mauricio Celedón présentée à la IX Edició del Festival Internacional de Teatre i Animació de Viladecans représentée en Espagne pour la première fois. Il s'agit d'une biographie en images sans aucun texte et accompagnée d'une musique rock. Le spectacle mettait en avant la souffrance d'Artaud dans l'asile mental et adoptait une esthétique proche de l'expressionnisme et du cirque basée sur le mouvement du corps (La Vanguardia, 02/07/1998:

76 Renommée salle de spectacle alternative à Madrid de 1988 à 2013. Actuellement, c'est le siège du Teatro del Barrio, dont l'acteur et dramaturge Alberto San Juan est le promoteur.

77 “[...] muy simple, muy elemental; pero al que no le falta ni poder de sugestión ni capacidad de comunicación”. Traduction française de l'auteure de cet article.

78 "La única posibilidad de que aparezca Artaud es la no interpretación, no intento adaptar su pensamiento, sus textos, sino atenerme a la letra estricta, a la ferocidad de su letra”. Traduction française de l'auteure de cet article. 
43). Quant à Sueños fugaces, il s'agit d'une production de Troysteatro mise en scène par Ernesto Rodríguez. La première se tient le 19 mars 2000 au grand amphithéâtre de la Universidad de La Laguna (Santa Cruz de Tenerife, Las Canarias). Nous n'en avons presque aucune donnée, si ce n'est sa trace dans le Centro de documentación teatral de Madrid, grâce à laquelle nous savons que le spectacle était construit à partir d'un texte à plusieurs créateurs intégrant des fragments d'Artaud.

L'année 2001 est une date spéciale en ce qui concerne la représentation théâtrale sur Artaud. Il est mis en scène par une troupe à renommée internationale, l'emblématique compagnie de théâtre du Berliner Ensemble, fondée par Bertold Brecht, et joué dans un théâtre très prestigieux de Madrid, le Teatro de la Abadia ${ }^{79}$. En fait, la compagnie présente, dans le cadre du festival d'automne, un spectacle autour de la figure d'Artaud, Artaud recuerda a Hitler y el Romanische Cafe $e^{80}$, dont la première se tient le 31 octobre. La troupe reprend cette production quelques années plus tard, en 2007, et la présente cette fois-ci au Teatre Lliure $^{81}$ de Barcelone le 5 mars. Le spectacle part d'un document: une lettre de huit lignes qu'Artaud écrivit en 1943 à Hitler dans laquelle il faisait référence à leur rencontre en 1932 dans un café de Berlin; un épisode dont on ne dispose d'aucune évidence. Le spectacle met justement en scène cette supposée rencontre entre Artaud et Hitler à travers le monologue du premier. Sur scène, un seul acteur interprète Artaud enfermé dans une cage en cristal qui représente l'asile de Rodez. La pièce aborde, d'une part, le thème de la folie et de la lucidité d'Artaud et, d'autre part, déploie un discours sur la théorie théâtrale de l'auteur (R.T., 01/11/2001: 36; Caruana, 01/11/2001: 61; Gómez, 01-11-2001: 44; Galindo, 01/11/2001: 27; A.J.N., 01/03/2007: 30; Ferré, 09-03-2007: 42) en soulignant le besoin d'un théâtre "cruel" et antibourgeois. En général, les critiques se limitent à présenter un spectacle dont la qualité semble garantie par la compagnie allemande sans en offrir une appréciation personnelle. Elles mettent en valeur la qualité indiscutable de la légendaire Berliner Ensemble et de l'acteur à renommée internationale Martin Wuttke avec "son impressionnant registre scénique" (Gómez, 01-11-2001: 44) qui devient dans le monologue "un robuste et électrique personnage" (Fondevila, 06-03-2007: 36). Il n'y a que la chronique de Joan-Anton Benach qui ne dissimule pas ses réticences aussi bien par rapport au texte de Peuckert qu'à la proposition scénique ou à l'interprétation:

Il est évident que Peuckert n'a pas organisé le saugrenu récit de sa si tourmentée créature sans une certaine logique interne. [...] Wuttke exécute une superbe exhibition de gestes, grimaces, hurlements, rugissements, acrobaties [...] Fantastique ! Parler de perfection, parler de correction serait pourtant question de psychiatrie, et donc de déter-

79 Théâtre emblématique et centre d'études et de création scénique dirigé par l'acteur et metteur en scène José Luis Gómez.

80 Monologue de Tom Peuckert, mise en scène de Paul Plamper, scénographie et costumes de Paul Lerchbaume et interprété par Martin Wuttke.

81 Théâtre très prestigieux créé en 1976 par un groupe de professionnels provenant du théâtre indépendant. C'est également un centre de création. 
miner la maladie précise du personnage. Il est bien connu que dans les interprétations folles, on prend brillamment des vessies pour des lanternes ${ }^{82}$ (Benach, 09/03/2007: 50).

Il est curieux de constater, si l'on s'en tient aux propos de Benach, que les approximations à la figure d'Artaud, même lorsque celles-ci sont réalisées par de compagnies de théâtre prestigieuses, n'arrivent pas à se défaire tout à fait du cliché artaudien, ce qui met en lumière la problématique de la mise en scène de l'auteur et la remet certainement en question.

En mars 2004, la compagnie Triángulo/Tritea, dirigée par Shía Arbulú, présente dans la Sala Triángulo de Madrid un nouveau spectacle, Artaud ahora (2004), monté à partir du texte d'Alfonso Pindado qui dit s'être inspiré des années d'Artaud à Rodez et du théâtre de la cruauté pour montrer "la peur et la valeur de la vie dans des situations extrêmes" (Pindado in Henríquez, 02/04/2004: 117) ${ }^{83}$. Située dans un asile mental, la pièce aborde la question de la folie en tout temps et situe Artaud au centre de la réflexion. Sur le plan esthétique, il faut faire mention des jeux de lumières et d'obscurité mis en œuvre et de la disposition des interprètes dans l'espace de la salle, le public presque encerclant la représentation; une approximation de la configuration de la scène proche de celle d'Artaud ${ }^{84}$. Le chroniqueur Miguel Ayanz parle d'un spectacle plein de douleur et de folie, et applaudit l'interprétation des acteurs, la fonctionnalité de la scénographie, la mise en scène d'Arbalú et une compagnie qui “se donne à fond dans une pièce frissonnante [...] l'une des propositions les plus intéressantes du panorama alternatif"85 (Ayanz, 27/03/2004: 47).

Un an plus tard, en mai 2005, voit le jour un autre spectacle, La Gran Pregunta. Misa de cuerpo presente, de la Compagnie Atropos et du metteur en scène argentin Gabriel Monedero, qui s'occupe également de la percussion. Créée à partir de textes d'Antonin Artaud le poème concerné, des lettres et d'autres documents —, la pièce récupère le caractère sacré du théâtre et du rituel, et stimule l'immersion du spectateur dans la représentation, les sensations à travers la musique et les odeurs. Monedero crée ainsi un montage qui joint la philosophie théâtrale d'Artaud au taoïsme et aux rites indigènes guaranis, lesquels sont projetés en scène afin que les spectateurs puissent vivre l'ambiance de cette célébration et y être intégrés (I.F., 26/05/2005: 71). La pièce offre également un parcours retraçant la vie d'Artaud — la

82 "Es evidente que Peuckert no organizó el disparatado relato de su atormentada criatura sin una cierta lógica. [...] Wuttke ejecuta una soberbia exhibición de gestos, muecas, alaridos, rugidos, acrobacias [...] ¡Fantástico! Hablar de perfección, hablar de corrección sería, no obstante, misión de psiquiatría, luego de diagnosticar la dolencia precisa del personaje. Es sabido que por las interpretaciones locas, corren gatos en vez de liebres que da gusto". Traduction française de l'auteure de cet article.

83 "El miedo y el valor de la vida en situaciones extremas". Traduction française de l'auteure de cet article.

84 Artaud propose une architecture autre de la salle établissant une nouvelle relation scène-public. Il s'agirait d'un lieu unique où le spectateur, placé au milieu de la salle, serait enveloppé par l'action. Voir Artaud, Antonin. 1964. Le théâtre et son double, Paris, Éditions Gallimard: 148-150.

85 "Lo dan todo en escena [...] una de las propuestas más interesantes en el panorama alternativo". Traduction française de l'auteure de cet article. 
mystique, la folie, la maladie, le surréalisme. Le mythe Artaud revient sur scène une fois de plus en suivant certains postulats de la théorie du théâtre de la cruauté.

Comme nous venons de le constater, depuis 1990 jusqu'en 2005, tous les spectacles abordent, d'une manière ou autre, la question de la folie et de la lucidité d'Artaud. Pour ce faire, dans la plupart des productions, on met en scène un épisode de la biographie de l'auteur. En effet, les événements de la vie d'Artaud occupent une place essentielle dans les spectacles de cette période, en particulier son séjour à l'asile de Rodez, point de départ pour problématiser sur l'aliénation mentale et la clairvoyance du poète, mais aussi pour déployer un discours sur ses théories théâtrales et exposer la pensée de l'auteur recueillie dans ses essais. D'ailleurs, le monologue s'impose dans plusieurs propositions scéniques autour de la figure d'Artaud, que ce soit chez les jeunes compagnies de théâtre ou parmi les professionnels et les troupes renommées: Artaud! El teatre de la crueltat (1991), Van Gogh: el suicidado de la sociedad (1992), Artaud, retrato feroz (1994) et Artaud recuerda a Hitler y el Romanische Café (2001). En outre, les créateurs entendent faire un théâtre selon les consignes données par l'auteur et essaient de les matérialiser sur scène dans tous les spectacles sans exception: théâtre sacré, rituel, corporel, etc. Nous sommes également témoins d'un phénomène: la naissance du suppôt artaudien qui est créateur, dramaturge et interprète. C'est le cas, par exemple, de Ramon Costa qui crée son monologue pour Spitteatre en 1991 et qui continue à le retravailler et à le représenter en public pendant treize ans, mais aussi du monologue de l'argentin Gorosco dans son Artaud, retrato feroz qu'il représente durant deux décennies.

\subsection{Dernières expériences: répétition et différence}

Les spectacles autour d'Artaud à partir de 2006 suivent à peu près les grandes lignes exposées dans les deux étapes antérieures. Cependant, après 37 ans d'expérience dans la représentation de l'auteur sur les planches, certains metteurs en scène tentent de s'approcher de la figure d'Artaud autrement en fuyant les stéréotypes avec plus ou moins de succès. Ainsi, en 2006, apparaît une œuvre qui mérite une attention spéciale. Le dramaturge Ózcar Galán écrit, en 2006, Artaud, rien de rien, où le titre déjà renvoie à deux personnalités du milieu parisien. La pièce, dont la première se tient le 22 juin 2006, est mise en scène par Xavier Castiñeira et représentée à la RESAD ${ }^{86}$ dans la Sala Valle-Inclán. Elle remporte le premier prix Villa Pinto de Textos Teatrales la même année et est publiée en 2012 chez Anagnórisis. Structurée en neuf scènes, la pièce met en œuvre 15 personnages, dont Artaud et Edith Piaf, situés dans la France occupée pendant la Seconde Guerre mondiale. L'action commence dans l'asile mental où Artaud est enfermé; puis, dans la plupart des scènes, elle se situe dans un bordel, pour revenir à l'asile lors des deux dernières scènes quand on évoque la mort d'Artaud. Néanmoins, le drame ne se centre ni sur la personne ni sur l'art de ces deux person-

86 Real Escuela Superior de Arte Dramático. 
nalités. En fait, Artaud devient un personnage de plus parmi d'autres et sa conduite fuit les stéréotypes de l'auteur auxquels on est habitués et qu'on a tant exploités dans toutes les productions abordées jusqu'à présent; pas de cris ni hurlements, Artaud s'exprime calmement au travers d'un langage commun, très éloigné de sa prose habituelle. Comme le souligne Pedro Víllora, "L'histoire de Artaud, rien de rien n'est pas la leur [celle d'Artaud et Piaf]. C'est l'histoire de gens qui n'ont pas besoin qu'on leur explique ce qu'est la cruauté et pour qui, s'il y a une vie, celle-ci existe en marge du théâtre" "V7 (Víllora in Ozkar Galán, 2007: 7-11).

C'est la guerre, la misère, la prostitution, ce qui prend ici le dessus. La dernière réplique de la pièce, proférée par Piaf après la mort d'Artaud, synthétise ce qui unit presque tous ces personnages en situation de fragilité: “La terre n'a pas été créée pour les races faibles" $" 88$ (Galán, 2012: 236). Dans Artaud, rien de rien, la présence d'Artaud, ainsi que celle d'Edith Piaf, aide à agglutiner tous ceux qui sont définis par l'exclusion, la fragilité et l'oppression.

En 2007, un autre spectacle voit le jour. Teatro Corsario présente, dans l'Espacio Fonseca de Salamanca, Pasión Artaud, qui, comme le nom l'indique, joint la passion du Christ avec celle d'Antonin Artaud. Le metteur en scène du spectacle, Fernando Urdiales, puise dans les processions de la Semaine Sainte espagnole, où il existe "une beauté chargée d'extrême violence", pour la relier aux textes d'Artaud. Selon la critique, dans Pasión Artaud, "il y a une violence dans les textes mais aussi une beauté poétique sublime" ${ }^{89}$ (ICAL, 02/06/2007: 57). L'union d'Artaud aux rituels de la Semaine Sainte accorde à ce spectacle une grande dose d'originalité et situe Artaud du côté des torturés de la société.

Plusieurs sont encore les spectacles autour de la figure d'Antonin Artaud dans ce début du siècle: 1. Un bisou pour Antonin Artaud (2008) de La Calba Teatre, un hommage à l'auteur mis en scène par Júlia del Solar et représenté à Barcelone à l'Ateneu Popular Nou Barris — proposition de théâtre expérimental —; 2. El poder de la sangre (2010), lecture dramatisée, le 16 décembre dans la Sociedad Cervantina de Madrid, d'un texte inspiré de Les Cenci d'Artaud, mise en scène de Sonia Sebastián; 3. Fuerza Oculta (un pensamiento de Antonin Artaud) (2010), production de El Caldero Teatro Estudio de Buenos Aires, réalisée et interprétée par l'argentin Daniel Lambertini et représentée à Madrid au Teatro de la Puerta Estrecha du 25 mars au 4 avril. Ce spectacle privilégie l'esthétique du rituel et est créé à partir de trois textes d'Artaud: Le théâtre et son double, Les Tarahumaras et Van Gogh. Le suicidé de la société; 4. Los Cenci (2013), mis en scène par Sonia Sebastián au Teatro Español de Madrid; 5. Hombres Bisagra (2014) de Matarile Teatro; 6. El teatre i la pesta (2015), monologue à partir du texte homonyme de l'auteur réalisé par Jordi Coca au Teatre Akadèmia

87 "La historia de Artaud, rien de rien no es la de ellos. Es la historia de unas gentes a quienes nadie tiene que explicar lo que es la crueldad y para quienes, de haber vida, la hay al margen del teatro". Traduction française de l'auteure de cet article.

88 "La tierra no ha sido creada para las razas débiles".

89 "Hay violencia en los textos y también una belleza poética sublime". Traduction française de l'auteure de cet article. 
de Barcelone; et 7. A propòsit d $\square$ Artaud (2016), le 26 avril au Teatre La Seca de Barcelone, lecture de ses poèmes dans le cadre du Festival Alcools, Poesia en escena 2016, réalisée par la performeur Clara Dorothee Hahn et les artistes Edgar Alemany et Gabriel Ventura.

Trois de ces productions méritent une attention particulière. La première, Los Cenci, mise en scène par Sonia Sebastián ${ }^{90}$, est représentée au Teatro Español ${ }^{91}$ de Madrid du 17 janvier au 3 mars 2013. Elle présente une version libre contemporaine, mais très respectueuse, du texte d'Artaud. Quant à la mise en scène, il existe une volonté manifeste de matérialiser sur scène certains aspects du théâtre de la cruauté. Sonia Sebastián met en place une scène polyvalente, de forts effets visuels et sonores, un mouvement des corps dans l'espace inspiré de la danse contemporaine et un jeu des acteurs ayant lieu à différentes hauteurs (López Rejas, 11/01/2013: 43; Rodríquez Marcos, 17/01/2013: 41; J.B., 18/01/2013: 44; Ayanz, 19/01/2013: 68; Villán, 27/01/2013: 52; Hidalgo, 01/02/2013: 44; Vallejo, 02/02/2013: 3). Le spectacle est applaudi par le public et la critique, qui exalte, en général, la plasticité des images, la mise en scène et le jeu des acteurs (ibid.). D'ailleurs, la pièce est représentée dans un théâtre emblématique de Madrid et elle fait l'objet de nombreuses critiques dans les journaux, ce qui montre que le spectacle bénéficia d'un retentissement considérable. Il s'agit, en effet, d'un spectacle sérieux fuyant les stéréotypes qui montre, comme disait Grotowski, que la cruauté, c'est aussi la rigueur (Artaud, 1972: 17). Il nous semble enfin que le montage de Sebastián a, en outre, la vertu d'avoir su garder et actualiser ce qui est universel dans la pièce d'Artaud, à savoir: la question des abus de pouvoir et le déchaînement des pulsions.

Le deuxième spectacle qui a retenu notre attention est Hombres bisagra (2014), conçu par Baltasar Patiño et Nacho Sanz, de la célèbre troupe galicienne Matarile Teatro, dirigée par Ana Valdés. La pièce reçoit le prix au spectacle le plus novateur du TAC XVI Festival Internacional de Valladolid 2015. Il s'agit d'un montage qui se situe à mi-chemin entre le concert (des percussions et des synthétiseurs primitifs) et la représentation théâtrale. Le spectacle débutait avec l'enregistrement radiophonique d'Antonin Artaud Pour en finir avec le jugement de Dieu et, sur la scène, une pancarte montrait le nom de l'auteur. Invoqué depuis le début, Artaud apparaissait au cours de tout le spectacle: des extraits de l'enregistrement radiophonique et de brefs fragments de Le théâtre et son double s'inséraient dans le déroulement du montage tels une revendication et adhésion esthétique. L'action scénique qui s'y déployait suivait certains préceptes d'Artaud explicités dans Le théâtre et son double (Becerra, 2014), une conception du théâtre présente depuis la création de la compagnie en 1986. Plasticité des corps, dimension poétique de la scène, des images, des sons, des lumières, des mouvements suggérant des situations, telle était la proposition scénique de Matarile Teatro.

La dernière production que nous aborderons ici est El teatre i la pesta, présentée au Teatre Akadèmia de Barcelone du 9 au 26 juillet 2015 dans le cadre du Festival Grec. Il s'agit d'une adaptation de la conférence d'Artaud à la Sorbonne en 1933, traduction de Joan Casas.

90 Sonia Sebastián s'était déjà rapprochée de ce texte d'Artaud lors de la lecture dramatisée en 2010 (cf. supra).

91 Théâtre public de Madrid. 
Le dramaturge et metteur en scène, Jordi Coca, transforme ce texte en monologue, joué par Esther Bové. Coca se centre ici sur le texte, qui prend le rôle protagoniste, et ne s'occupe pas de la figure de l'auteur: "Je ne voulais pas un acteur jouant Artaud avec tous ses clichés, ses cris et son hystérisme"92 (Coca in Fernández, 09/07/2015: 58-59). Le spectacle s'éloigne ainsi des montages mettant en scène Artaud lui-même, sa vie et son délire, et des mises en scène ou du jeu à facture artaudienne. La scénographie et l'actrice, toutes deux d'une grande élégance, subissent une décomposition progressive, comme atteintes par la peste, laquelle, comme le souligne le texte, “est bienfaisante, car poussant les hommes à se voir tels qu'ils sont, elle fait tomber le masque, elle découvre le mensonge, la veulerie, la bassesse, la tartuferie" (Artaud, 1964: 46). Le spectacle, d'une profondeur et d'une subtilité remarquables, reçoit une bonne critique, bien que limitée, et il est accueilli chaleureusement par le public dans la petite salle du Teatre Akadèmia.

Comme nous venons de le constater, les spectacles réalisés entre 2006 et 2016 reproduisent grand nombre des sujets abordés dans les productions précédentes. Plusieurs se centrent toujours sur la vie d'Artaud - l'asile de Rodez, la souffrance de l'auteur, l'exclusion - d'autres privilégient ses textes et consacrent tout le spectacle au déploiement de sa pensée et de son écriture sur scène, et il y en a aussi certains où Artaud devient un étendard en tant que déclaration de principes de la création théâtrale. Nous avons aussi été témoins de l'intérêt porté à la pièce de l'auteur, Les Cenci, Sonia Sebastián la mettant en scène à Madrid en 2013, soit 44 ans après la première représentation de ce texte en Espagne. Néanmoins, il est à noter que quelques-uns de ces spectacles s'éloignent des clichés créés autour de l'auteur ainsi que des mises en scène et des interprétations folles, et tentent d'imaginer une nouvelle manière de penser et de représenter Artaud. C'est le cas des trois dernières productions examinées ici, mais aussi de la pièce de Ózcar Galán, où Artaud devient, pour la première fois, un personnage de fiction d'une nouvelle écriture dramatique lui conférant un nouveau rôle dans le spectacle. Dans tous les cas sans exception, la mise en scène se veut artaudienne, ce qui a été une constante dans toutes les productions abordées dans cette étude. Il faut enfin souligner que ces productions se distinguent, en général, par leur qualité, ce qui est reconnu de manière unanime et apprécié par la critique et le public.

\section{Conclusion}

Si nous jetons un coup d'œil aux représentations qui se sont succédé en Espagne de et sur Artaud depuis 1969 jusqu'à nos jours, nous apercevons quelques constantes. En premier lieu, il existe toujours la volonté de matérialiser sur scène les théories théâtrales de l'auteur. Évidemment, chacune des productions a mené à terme ce dessein partiellement en privilégiant tel ou tel principe: le déploiement des langages non verbaux sur scène, le caractère

92 "No quería a un actor que imitara a Artaud con todos sus tópicos, gritos e histerismo". Traduction française de l'auteure de cet article. 
rituel et sacré du théâtre, la cruauté, etc. En effet, le théâtre visé par Artaud dans sa globalité est irréalisable. Derrida avait déjà affirmé que la fidélité absolue à Artaud était impossible et énumérait la liste de ce qui était contraire à sa conception théâtrale; parmi les trahisons: "tout théâtre non sacré [...], tout théâtre privilégiant la parole [...], tout théâtre de la distanciation, tout théâtre [...] cherchant à délivrer un message"(1967: 358-361). Les montages réalisés en Espagne ont donc tenté d'être fidèles aux postulats d'Artaud sans pouvoir pourtant éviter de le trahir d'une manière ou une autre. En fait, il s'agit de spectacles, en général, qui veulent donner à connaître l'auteur et ses textes, et qui privilégient, par conséquent, un "théâtre du verbe $[\ldots]$ et de communication [...] cherchant à transmettre un contenu"(ibid.), ce qui est étranger au théâtre de la cruauté, comme le signale Derrida. Cependant, malgré l'impossibilité de faire un théâtre artaudien en termes absolus, le projet Artaud a inspiré la mise en scène de toutes les productions abordées dans cette étude et a donc représenté une fenêtre certaine à une nouvelle conception du théâtre et de la représentation. Il faudrait pourtant faire une dernière remarque à ce propos. Si les compagnies et metteurs en scène, pour représenter Artaud, ont voulu mettre en œuvre un théâtre révolutionnaire dans la forme et le contenu, l'un étant condition requise de l'autre, parfois, sur la scène espagnole, sa matérialisation reste quelque peu en surface et n'atteint pas la transcendance qui est censée l'accompagner. Cela n'est pas exclusif de la scène espagnole. En fait, Grotowski avait dénoncé, à propos d'un grand nombre de spectacles de l'avant-garde théâtrale d'Occident des années 60, une mise en œuvre des théories artaudiennes qui était à ses yeux déplorable: "ces pièces chaotiques, avortées, pleines d'une supposée cruauté [...] qui montrent un manque de maîtrise du métier, multiplicité d'erreurs et de facilités et qui ne sont qu'extérieurement violentes"(in Artaud, 1972, 7-8). S'il est vrai qu'il a pu y avoir une banalisation de l'auteur et de ses théories aussi en Espagne, il ne faut pourtant pas sous-estimer l'importance de ces expériences théâtrales. Les théories d'Artaud ont certainement animé et orienté les metteurs en scène espagnols en leur permettant de se frayer un chemin vers un renouveau des langages scéniques. De plus, ces mises en scène se perfectionnent au cours des années; des premières ébauches enthousiastes, mais pas toujours très réussies, à la subtilité de certaines productions, tout un chemin a été fait et une nouvelle manière de penser le théâtre a pris racine. En outre, ces préceptes théâtraux sont répandus et s'appliquent à d'autres auteurs, sujets et mises en scène et partagent les planches avec la création théâtrale plus traditionnelle. En deuxième lieu, comme on l'a indiqué en amont, la représentation théâtrale en Espagne a donné à connaître la pensée d'Artaud à travers un grand nombre de textes et d'essais de l'auteur mis en scène: Le théâtre de la cruauté; Van Gogh: le suicidé de la société; Le théâtre et la peste; Pour en finir avec le jugement de Dieu; Héliogabale ou l'anarchiste couronné ou Fragments d'un journal d'enfer entre autres; ainsi que sa pièce Les Cenci. La poésie de l'auteur, en revanche, n'a pas bénéficié du même retentissement sur les planches. En effet, l'importance de la représentation théâtrale pour la vulgarisation de l'œuvre d'Antonin Artaud n'est pas négligeable, bien au contraire; la portée 
de ces initiatives est décisive et mérite une reconnaissance toute particulière. Cependant, ces représentations théâtrales ont eu lieu, à quelques exceptions près, dans des salles de théâtre alternatif et n'atteignent pas, en général, le grand public. Comme nous l'avons souligné au début de cette étude, ce genre de spectacles fait partie d'un théâtre minoritaire et non conventionnel ayant comme public des hommes et des femmes de théâtre, des connaisseurs et des intellectuels. En dernier lieu, il faut souligner que si la scène a divulgué l'écriture de l'auteur et favorisé la mise en place d'un théâtre autre, la figure d'Artaud a été pourtant usée à l'extrême. En effet, l'auteur devient souvent un personnage très stéréotypé: le fou, le malade, le torturé.. Quelques-uns en font même une source inépuisable: les productions de Jorge Gorosko et de Ramon Costa en sont une preuve. Qui plus est, l'interprétation du personnage Artaud abuse en excès des tics qui lui sont associés: son hystérie, ses hurlements, ses exclamations. La vie d'Artaud se transforme ainsi en sujet de fiction de grand nombre de spectacles: l'asile de Rodez et la souffrance, un homme démembré, des spasmes et des convulsions; Artaud devient fatalement cliché. Et cela n'est pas sans conséquences, car l'exploitation récurrente de ce lieu commun dépouille, à la longue, la pensée de l'auteur et son œuvre de l'épaisseur littéraire et philosophique qui les caractérise. Dans ce sens, pourrait-on donc en conclure qu'en Espagne la réception d'Artaud sur les planches n'évolue pas et qu'elle n'a su échapper à une certaine mystification du personnage? Autant l'affirmation que son contraire sont valables. Il y a, il est vrai, quelques productions scéniques qui s'écartent de cette vision et qui adoptent des approches plus novatrices, surtout à partir de l'année 2006, ce qui correspond, d'une certaine manière, à une volonté de sortir des sentiers battus. Artaud, rien de rien d'Oskar Galán, Les Cenci de Sonia Sebastián ou El teatre i la pesta de Jordi Coca en sont quelques exemples. Il $\mathrm{y}$ a donc, chez certains créateurs, une prise de conscience de cette saturation et banalisation du personnage sur scène. Mais il nous semble, en même temps, qu'il est difficile de pouvoir y échapper complètement. La revue situationniste Potlach avait déjà dénoncé en 1955 la mystification de l'auteur lorsqu'elle affirmait que l'attention avait "été détournée il y a quelques années vers le cadavre surfait d'Antonin Artaud" (Debord, G-E. et Wolman, G.J., 1955). Il y a eu, effectivement, une certaine glorification de l'auteur, en Espagne et ailleurs, qui a entraîné un appauvrissement dans le regard porté sur Antonin Artaud. Et cela se matérialise dans des approches qui souvent défigurent et réduisent le personnage à une stylisation simpliste et stéréotypée. En fait, Durozoi affirme qu'Artaud ne peut être source d'inspiration parce qu'il devient aussitôt "une tentation au plagiat, ou une invitation à la trahison involontaire - sans que l'on puisse dire où est le pire, du calque et de la répétition ou de la déformation" (1972: 246). Serions-nous alors face à une impasse incontournable? Il se peut. En tout cas, il semble certain que les auteurs et metteurs en scène qui souhaitent représenter Artaud dorénavant devraient remettre en question un modèle de représentation qui est quelque peu épuisé à l'heure actuelle. Il leur faudrait sans doute explorer de nouvelles voies, sans faire d'Artaud une formule ou un gourou. Il leur faudrait trouver, par leurs propres moyens, leur langage 
scénique à eux, oser risquer et entrer en terrains inconnus. N'est-ce pas, en fait, la grande leçon que l'on puisse tirer d'Antonin Artaud?

\section{Références bibliographiques}

A. J. N. 01/03/2007. "Los diálogos impossibles entre Artaud y Hitler” in El Periódico, 30.

Anguera, Joan. 1996. "Crònica particular d'una relació amb el Sr. Antonin Artaud" in $A s-$ saig de teatre: revista de l'Associació d'Investigació i Experimentació Teatral, nº4, 31-35.

Aragon, Louis. 15 juillet 1925. "Fragments d'une conférence" in La Révolution surréaliste, $\mathrm{n}^{\circ} 4$, Editions de la Nouvelle Revue Française, 23-24.

Artal, Rosa María. 21/05/1980. "Montaje con textos de Antonin Artaud" in El País, < https://elpais.com/diario/1980/05/21/cultura/327708018_850215.htm >.

Artaud, Antonin. 1956-1957. Euvres Complètes (Vol. IV). Paris, Gallimard.

Artaud, Antonin. 1964. Le théâtre et son double. Paris, Éditions Gallimard.

AyAnz, Miguel. 27/03/2004. "Artaud ahora, una casa repleta de dolor y locura” in La Razón, 47.

Ayanz, Miguel. 19/01/2013. “Artaud, pura crueldad” in La Razón, 68.

Becerra, Alfonso. 17/10/2014. "Cosmogonía teatral y Hombres Bisagra” in Artezblai: el periódico de las artes escénicas $<\mathrm{http}$ ://www.artezblai.com/artezblai/cosmogonia-teatral-y-hombres-bisagra.html $>$.

BenaCH, Joan-Anton. 04/07/2001. "Un discreto entre malditos” in La Vanguardia, 43.

BenACH, Joan-Anton. 09/03/2007. "El delirio hitleriano de Artaud" in La Vanguardia, 52.

Bradby, David. 1990. Le Théâtre Français Contemporain 1940-1980. Paris. Presses Universitaires de Lille.

Caruana, Pablo. 01/11/2001. "El Berliner expande la peste de Artaud en la Abadía" in La Razón, 61.

CASCón I RAmoneda, Carles. 25/06/1991. "Artaud ressuscità de nou al Casal Pere Quart" in Sabadell, 32.

Dalmau, Helios. 05/11/1987. "Espectacle sobre poemes d'Artaud” in Diario de Barcelona, 24.

Debord, Guy-Ernest \& Gil J. Wolman. 1955 (9 septembre). “Pourquoi le lettrisme?” in Potlatch, $\mathrm{n}^{\circ}$ 22. $<$ http://debordiana.chez.com/francais/potlatch22.htm\#precieux $>$.

De La Hera, Alberto. 13/02/93. "Habilidad y maestría" in Ya.

DerridA, Jacques. 1967. L'écriture et la différence. Paris, Éditions du Seuil. 
De Sagarra, Joan. 07/11/1987. "Homenaje a Artaud” in El País, 34.

Durozor, Gérard. 1972. Artaud: l'aliénation et la folie. Paris, Larousse Université, Collection Thèmes et Textes.

Ferré, Teresa. 09/03/2007. "El crit” in El Punt, 42.

Fondevila, Santiago. 6/3/2007. “Artaud habla con Hitler en el Lliure y Oriol Broggi toma la Biblioteca de Catalunya" in La Vanguardia, 36.

Font, Domenec. 1972. “¿La nueva era Artaud?” in Primer Acto 148, 5-6.

Galán, Oskar. 2007. Unamuno, ad altior natus sum. Bilbao. Artezblai.

GalÁn, Oskar. 2012. Artaud, rien de rien. Editorial Anagnórisis. $<$ http://www.anagnorisis. es/wp-content/themes/journalized/images/libros/Artaud,_rien_de_rien.pdf >.

Galindo, Carlos. 01/11/2001. "El Berliner Ensemble presenta en el Festival de Otoño la figura de Artaud" in $A B C, 27$.

GARCía LoRenzo, Luciano. 1999. Aproximación al teatro español universitario (TEU). Madrid, Consejo Superior de Investigaciones científicas.

Gomez Picazo, Elías. 21/04/1970. "Nacional de cámara: 'Los Cenci', de Artaud, por el T.U.C. de Barcelona" in Madrid, 21.

Gómez, Marta. 01/11/2001. "El arte total de Artaud llega al Teatro de la Abadía con el Berliner Ensemble" in Diario 16, 44.

Grotowski, Jerzy. 1972. "Artaud y el teatro de la crueldad" in ARTAUD, Antonin. Tres piezas cortas. Madrid, Fundamentos, 7-18.

FERnÁndez, Imma. 09/07/2015. “Jordi Coca rescata al 'apestado' Artaud” in El Periódico, 58-59.

Henríquez, José. 02/04/2004. “Triángulo apuesta por la continuidad” in Guía del Ocio, 117.

Hidalgo, Manuel. 01/02/2013. “Antonin Artaud. Montaje de 'Los Cenci’” en el Teatro Español de Madrid. El loco del teatro" in El Mundo, 44.

ICAL. 02/06/2007. "Teatro Corsario celebra su 25 aniversario con 'Pasión Artaud"” in Tribuna, 57.

I. F. 26/05/2005. "Misa por Antonin Artaud" in El Cultural. El Mundo, 71.

INTERINO. 11/06/1982. "Descenso a los infiernos" in $A B C, 69$.

J.B. 18/01/2013. "Los Cenci. Rebelión contra la crueldad” in $A B C, 44$.

Kustow, Michael. 1965. "Sur les traces d'Artaud” in Esprit, no 338 (5), 958-963.

LÁzaro Carreter, Fernando. 1973. "Artaud y el teatro contemporáneo" in Primer Acto, n 159-160, 12-21 
López ReJAS, Javier. 11/01/2013. “Artaud asalta las butacas del Español” in El Cultural, 43. López SANCho, Lorenzo. 22/04/1970. "Antonin Artaud ('Los Cenci'), en teatro de cámara” in $A B C, 91$.

Lladó I Vilaseca, Jordi (2002). Ramon Vinyes i el teatre (1904-1939). Thèse doctorale, Universitat Autònoma de Barcelona.

Marquerie, Alfredo. 20/04/1970. "Estreno de 'Los Cenci', de Artaud, por el T.U.C., en el Marquina" in Pueblo.

Martinez Perez, Antonia. 1987. "Valoración e influencia de las teorías dramáticas de Artaud" in Anales de Filología Francesa, n² 2, 57-77.

Muñoz CÁliz, Berta. 2005. El teatro crítico español durante el franquismo, visto por sus censores. Madrid, Fundación Universitaria Española (Col. "Tesis Doctorales cum Laude", Serie Literatura, núm. 31).

MuÑoz CÁliz, Berta. 2007. "El teatro silenciado por la dictadura franquista" in Per Abbat. Boletín Filológico de Actualización Académica y Didáctica, 3, 85-96.

Non Signé. 1969. “Artaud a Jouvet” in Primer Acto, n 108, 10-11.

Non Signé. 1970. “Festival Palma - 69 de Teatro Universitario” in Yorick, nº 38, 63-64.

Non Signé. 20/04/1970. “Marquina (T.N.C. Y E.): 'Los Cenci', de Antonin Artaud” in Informaciones, 27.

Non Signé. 21-04-1970. "Estreno de 'Los Cenci', de Artaud, en el teatro Marquina” in Arriba, 18.

Non Signé. 12/05/1971. “Obras teatrales aprobadas por la Junta de Apreciación” in La Vanguardia, 55

Non Signé. 20/06/1991. "Un altre Artaud, si us plau” in Sabadell, 37.

Non Signé. 02/07/1998. “Cincuenta y dos compañías inundan de teatro las calles de Viladecans" in La Vanguardia, 43.

NON SIGNÉ. 13/05/2004. "El monòleg d'Artaud viatjarà a Vic aquest mes" in Diari de Sabadell, 25.

Non Signé. 04/11/2009. “Cambaleo lleva a escena 'Entre los paisajes' de Antonio Fernández Lera" in Artezblai. < http://www.artezblai.com/artezblai/cambaleo-lleva-a-escena-entre-los-paisajes-de-antonio-fernandez-lera.html >.

Palau I Fabre, Josep. 1948 (février), “Antonin Artaud, o la invitació a la follia” in Ariel, $\mathrm{n}^{\circ}$ $15,9-10$.

Palau I Fabre, Josep. 1976. Antonin Artaud: la revolta del teatre modern, Barcelona, Edicions 62.

RANCIÈRE, Jacques. 2008. Le spectateur émancipé. Paris, La fabrique éditions. 
RodríQuez Marcos, Javier. 17/01/2013. "Nunca pise un charco de sangre” in El País, 41.

R. T. 01/11/2001. "Martin Wuttke y el Berliner se adentran en el delirio de Artaud" in El País, 36.

Rubio, Carme. 2002. "L'activitat teatral a Vic a partir de la postguerra" in $A U S A \cdot X X \cdot 148$ 149, 221-243.

Torres, Rosana. 07-10-1994. "El actor Jorge Gorosco inaugura un teatro con la misma obra que interpreta desde hace 20 años" in El País. <https://elpais.com/diario/1994/10/07/madrid/781532692_850215.html >.

VAliño, Xavier. 20/12/1985. "Teatro Corsario y Qloaca Letal en la cárcel” in El Norte de Castilla, 54.

VAllejo, Javier. 02/02/2013. “Cuando Artaud resucitó, el Papa seguía allí” in El País, 3.

Villán, Javier. 06/11/1992. “Alternativas en el Festival de Otoño” in El Mundo, 48.

Villán, Javier. 27/01/2013. "Bienvenidos a la crueldad de Artaud” in El Mundo, 52.

Vinyes, Ramon. 1932 (29/X/1932). Teatre Català, no 5, 70-71. 